\title{
Food storage in a changing world: implications of climate change for food-caching species
}

\author{
Alex O. Sutton ${ }^{*}$, Dan Strickland ${ }^{2}$ and D. Ryan Norris $^{1}$
}

\begin{abstract}
Food caching is a behavioural strategy used by a wide range of animals to store food for future use. When food is stored, it is susceptible to environmental conditions that can lead to spoilage via microbial proliferation or physical and chemical processes. Given that the nutrition gained from consuming cached food will almost always be less than consuming it immediately upon capture, the degree of degradation will play a central role in determining the ecological threshold at which caching is no longer profitable. Our framework proposes that the degree of susceptibility among caching species is based primarily on the duration of storage, and the perishability of stored food. We first summarize the degree of susceptibility of 203 vertebrate caching species. Thirty-eight percent (38\%) of these species are long-term cachers (>10 days) but only $2 \%$ are both long-term cachers and store highly perishable food. We then integrate insights from the fields of applied food science and plant biology to outline potential mechanisms by which climate change may influence food-caching species. Four climatic factors (temperature, number of freeze-thaw events, deep-freeze events and humidity) have been shown to affect the degradation of food consumed by humans and are also expected to influence the quality of perishable food cached in the wild. Temperature and moisture are likely important factors influencing seemingly nonperishable seeds. Although we are able to provide broad classifications for caching species at risk of climate change, an improved understanding of how environmental conditions affect the quality and persistence of cached food may allow us to better predict the impact of changing climatic conditions on the fitness of food-caching animals.
\end{abstract}

Keywords: Deep-freeze, Environmental change, Food caching, Food degradation, Food science, Freeze-thaw cycle

\section{Background}

Evidence suggests that climate change is influencing a wide range of biological phenomena, including species distributions [33, 58, 99], population abundance [48, 54], and rates of extinction $[26,73]$. Such studies provide important documentation of the potentially serious impacts of climate change on natural systems but, with few exceptions (e.g. $[18,120,171,178])$, fail to identify the proximate mechanisms by which climate change has influenced fitness and population growth rates [25] and, by extension, community structure [108, 121, 172, 175]. Identifying proximate mechanisms is important for the development of predictive frameworks, permitting an

\footnotetext{
* Correspondence: asutto01@uoguelph.ca

'Department of Integrative Biology, University of Guelph, 50 Stone Rd East, Guelph, ON N1G 2W1, Canada

Full list of author information is available at the end of the article
}

evaluation of the susceptibility of different species to long-term changes in the environment [19].

Here, we seek to develop such a framework for foodcaching animals by integrating insights from the fields of food science and plant biology with knowledge of foodcaching behaviours and preferences. Food caching is a widespread behavioural adaptation used primarily by non-migratory species to store food for future use during periods of low resource availability or uncertainty [164]. Once a food item is cached, it is exposed to environmental conditions that can either maintain or degrade its quality over time. Furthermore, the degree of degradation may also depend on a variety of factors, including food type, the duration of exposure and the location where food is stored. Although these factors are well studied in the field of food science, they have not been considered in the context of climate change and caching species. 
Our motivation for understanding the mechanisms behind environmental degradation of cached food stems from our long-term research on a declining population of Gray Jays (Perisoreus canadensis) at the southern edge of their range [32, 110, 155, 171, 176]. Gray Jays cache a wide range of perishable food items during the late summer and fall and use this food for over-winter survival and late-winter breeding [154]. Waite and Strickland [171]) proposed that warmer fall temperatures may be leading to the degradation of cached food, which then carries over to influence breeding success. They presented some correlational support for the 'hoard-rot hypothesis' but the effect of fall temperatures on reproductive success was relatively small compared to the steep population declines and there was only weak evidence that fall temperatures increased over the period in which Gray Jays declined. Additionally, a recent experiment using simulated caches did not find a consistent effect of warmer fall temperatures on food quality [140]. Sechley et al. [140])) did, however, suggest that temperature thresholds, such as the presence or absence of deep freeze events, could be driving observed differences in the degree of food preservation over a latitudinal gradient. These lines of evidence lead us to speculate that (a) the persistence of stored food may be influenced by more than a simple linear relationship with temperature and (b) that a deeper understanding of how climate influences food quality may be gained by drawing upon applied research related to the degradation of food stored by humans.

Our three primary goals in this paper are to (1) place the potential effects of climate change on cached food quality in a broader context of the costs and benefits of caching, (2) introduce a framework, based on variation in caching behaviour across species and in the types of food they store, for assessing their susceptibility to climate change and, (3) use insights from the fields of food science and plant biology to identify environmental conditions that could contribute to the degradation or preservation of cached food in the wild. We anticipate that these new perspectives will stimulate future research on a wider range of caching species and improve our ability to understand the potential effects that climate change may have on this subset of animals.

\section{Relating the potential effects of climate change to the costs and benefits of caching}

To put the potential effects of climate change into a broader context, we consider a simple cost-benefit equation [4] in which the fitness (considered here as nutritional gain), $F$, of a caching species can be estimated by $F=G p-C$, where $G$ is the fitness (or nutrition) gained by eating a cached food item at a future date, $p$ is the probability that that food item is retrieved, and $C$ is the cost of deferring consumption of that food item (in other words, the nutrition lost from not consuming it upon capture). Andersson and Krebs [4]argued that, if $G p>C$, then caching would evolve. However, in theory [this principle could also be used to assess how the costs and benefits of caching may change over ecological time and this will be particularly relevant under rapidly changing environmental conditions.

Of course, many species-specific factors will influence $G, p$, and $C$. Past studies have primarily focused on determining what drives $\mathrm{p}$, for example, by quantifying the frequency of conspecific and heterospecific competitors pilfering cached food $[81,83,167]$ and estimating the cognitive ability of a species to recover stored food at a future date $[65,105,124,125]$. In most cases (particularly for species that cache perishable food), the nutrition gained from consuming a cached item will almost always less than consuming it immediately upon acquisition and that this is due, in large part, to the degradation of cached food over time. The rate at which a cached food item will degrade is dependent on duration for which it is stored and the type of food that is stored (see detailed discussion in section below).

As an example, we use this equation to estimate the effect of different climatic conditions on caching in Gray Jays. To estimate $G$, we use data from Sechley et al. [140] who determined the caloric value of mealworms experimentally cached over a typical storage-retrieval period (fall-winter) at two locations with different climatic conditions. Mealworms are an appropriate food item to use for estimating cached food degradation in Gray Jays because this species only stores perishable food, and arthropods constitute a significant portion of their diet [154]. In the middle of the range in Cochrane Ontario, where temperatures rarely go above freezing beginning in November, mealworms retrieved in March were an average of $0.977 \mathrm{kcal}$, whereas at the southern edge of their range in Algonquin Park, where temperatures are rarely below zero until November, the average caloric value of mealworms stored over the same period was $0.663 \mathrm{kcal}$. For $p$, we used estimates of cache retrieval from two nutcrackers $(0.84$; Nucifraga columbiana; [160], Nucifraga caryocatactes; [70]). Similar to Gray Jays, both species rely on cached food for both over-winter survival and reproduction and, therefore, likely have relatively accurate spatial memories [8] The cost of caching, $C$, can be estimated as the energy it takes an individual to cache a food item and find an additional item that is of equal nutritional quality to the item that was cached. Because the energetic cost of foraging is not known for Gray Jays, we estimated $C$ by taking an estimate of the daily energetic requirement of an individual Gray Jay (47 kcal; [141]) and multiplying it by the proportion of the day an individual would take to 
find a new food item $(24 \mathrm{hrs} / 10 \mathrm{~min}=0.007)$ resulting in $C=0.33 \mathrm{kcal}$. Using these values, the nutrition gained from caching food at the more southern site is estimated to be $>50 \%$ lower $(0.23 \mathrm{kcal})$ compared to the more northern site $(0.50 \mathrm{kcal})$. Given temperatures in Algonquin were similar to the Cochrane as early as 1990 [139], this suggests that Gray Jays in Algonquin park have experienced a significant decline in nutritional quality of cached food. Of course, these are crude estimates but they do serve as an example of how this equation can be used to assess the influence of environmental conditions on nutritional benefits of caching.

\section{Predicting the susceptibility of food-caching species to climate change}

Caching behaviour is widely distributed across 30 families of mammals and 15 families of birds and is believed to have evolved independently numerous times within each taxa [30, 57, 145, 164]. Given the repeated, independent evolution of caching behaviour, it is perhaps no surprise that there is also a wide diversity of caching behaviours and types of food that are cached ([164]; Table 1). We believe that the degree to which cached food is influenced by climate change depends primarily on three major axes of variation that we discuss in detail below. In order of importance they are: 1) the duration of time that food is stored, 2) the type of food that is cached, and 3) the location where food is stored (Fig. 1).

\section{Duration of food storage}

Caching species can generally be divided into two classes based on cache duration. The first is 'short-term cachers' that can be characterized by temporally overlapping and episodic caching and recovery events [164]. These species are primarily motivated by present and/or imminent uncertainty in resource availability and will typically cache food for no longer than 10 days before retrieval. One type of caching species that falls into this class are single-item-surplus cachers that cannot consume all of a prey item in a single sitting and, therefore, attempt to protect the remaining portion of food for future feeding bouts (e.g. Tigers, Panthera tigris; [138]). A second type of short-term cacher stores food to protect it from heterospecific and conspecific competitors. For example, Leopards will bring carcasses into trees in order to protect their kills from Lions and Hyenas [35, 39]. Barbados Green Monkeys (Cercopithecus aethiops) have been observed to cache food for short periods of time in order to prevent conspecific competitors from accessing their food [15]. A third type of short-term cacher are 'insurance' cachers, such as parids [142] and mustelids [146], who cache food items for hours or days as an apparent hedge against present or imminent uncertainty in the environment.
The second class of caching species is long-term cachers' that are characterized by distinct, nonoverlapping storage and recovery periods that are $>10$ days but are more typically separated by two or more seasons [164]. Long-term cachers store food as a response to a certain lack of food in the future. Species in this class typically engage in intense periods of caching, usually in the late summer or fall $[31,66,154,164]$ and are followed by intervals of limited resources (e.g. winter) during which caches are retrieved. Food stored by long-term cachers will be more susceptible to environmental change simply because it is exposed to the environment for longer periods compared to food stored by short-term cachers. Long-term storage also increases the probability that food will be stored during transitional periods between seasons that are characterized by large fluctuations in environmental conditions that can negatively affect food quality. For example, late summer and fall storage exposes items to potentially damaging freeze-thaw cycles as the year transitions into winter.

Latitude and altitude play an important role in determining the duration that food is stored, particularly for long-term cachers. At high latitudes, periods of limited resources are longer, which means more food must be cached over a comparatively shorter time period [24, 123]. This could imply that populations at higher latitudes are more susceptible to changing climatic conditions because of the presumably higher reliance on cached food compared to populations at lower latitudes. Similarly, along elevation gradients, high elevation population could be at greater risk due to an increased reliance on cached food [168]. Climate warming could benefit caching species by prolonging food availability during the storage season or by reducing the length of low-resource periods in which cached food is relied upon. Both of these examples highlight how caching behaviour changes over temperature gradients, thus, it will be important to consider how changing climatic patterns influence not only food once it is cached, but also how it could influence caching decisions.

\section{Type of food stored}

A wide variety of food is cached by wild animals $[29,145,164,166]$ and this variation will impact how susceptibility it may be to climate change. At the broadest level, the distinction can be made between perishable and non-perishable food items. Food perishability is primarily a function of its water content, which dictates not only how food will respond to freezing temperatures but also the growth rate of bacteria [64, 96, 102]. Although some species store exclusively perishable (e.g. carnivores, such as canids, felids and raptors; [27, 100], and Gray Jays; [154]) or non-perishable (e.g. tree squirrels Sciurus spp. and new world mice Peromyscus spp.); [104, 164]) food, many other species store both types of food. For example, 
Table 1 A summary of caching behaviour of 203 vertebrates (adopted and updated from [164]). Caching behaviour includes duration of food storage, the perishability and type of food cached, and the location where food is stored are included within the table. In many cases, information on each of these three categories represent a 'best estimate', as much of the literature consists of anecdotal reports or natural history observations. All species were assigned a susceptibility score from 1-9 based on variation in two major aspects of caching behaviour: duration and food perishability (see text for details). Susceptibility to climate change was predicted to be lowest for short-term cachers of low perishable food item (1) and highest for long-term cachers of perishable food items (9). More research is needed to understand how location of storage may influence degradation of cached food and was, therefore, not included in the susceptibility score

\begin{tabular}{|c|c|c|c|c|c|}
\hline Species & Duration & Perishability & Food cached & Placement & Susceptibility \\
\hline Jackdaw & S & Low & $\mathrm{E}, \mathrm{N}, \mathrm{Mi}, \mathrm{SM}$ & Arboreal Cavity & 1 \\
\hline \multicolumn{6}{|l|}{ Corvus monedula } \\
\hline Red tree vole & S & Low & WV & Arboreal Cavity & 1 \\
\hline \multicolumn{6}{|l|}{ Arborimus longicaudus } \\
\hline Black-capped chickadee & S & Low & I, N, S & Arboreal Surface & 1 \\
\hline \multicolumn{6}{|l|}{ Parus atricapillus } \\
\hline Boreal chickadee & S & Low & $\mathrm{N}, \mathrm{S}$ & Arboreal Surface & 1 \\
\hline \multicolumn{6}{|l|}{ Parus hudsonicus } \\
\hline Brown-headed nuthatch & S & Low & I, S & Arboreal Surface & 1 \\
\hline \multicolumn{6}{|l|}{ Sitta pusilla } \\
\hline Coal tit & $\mathrm{S}$ & Low & । & Arboreal Surface & 1 \\
\hline \multicolumn{6}{|l|}{ Parus ater } \\
\hline Eurasian nuthatch & $\mathrm{S}$ & Low & $\mathrm{N}, \mathrm{S}$ & Arboreal Surface & 1 \\
\hline \multicolumn{6}{|l|}{ Sitta europaea } \\
\hline Marsh tit & $\mathrm{S}$ & Low & $S$ & Arboreal Surface & 1 \\
\hline \multicolumn{6}{|l|}{ Parus palustris } \\
\hline Pygmy nuthatch & $S$ & Low & I, S & Arboreal Surface & 1 \\
\hline \multicolumn{6}{|l|}{ Sitta pygmaea } \\
\hline Red-breasted nuthatch & S & Low & $\mathrm{N}, \mathrm{S}$ & Arboreal Surface & 1 \\
\hline \multicolumn{6}{|l|}{ Sitta canadensis } \\
\hline Siberian tit & S & Low & S & Arboreal Surface & 1 \\
\hline \multicolumn{6}{|l|}{ Parus cinctus } \\
\hline Tufted titmouse & S & Low & $\mathrm{N}, \mathrm{S}$ & Arboreal Surface & 1 \\
\hline \multicolumn{6}{|l|}{ Parus bicolor } \\
\hline White-breasted nuthatch & $S$ & Low & $\mathrm{N}, \mathrm{S}$ & Arboreal Surface & 1 \\
\hline \multicolumn{6}{|l|}{ Sitta carolinensis } \\
\hline Large Japanese field mouse & S & Low & $N, S$ & Subterranean Ground & 1 \\
\hline \multicolumn{6}{|l|}{ Apodemus speciosus } \\
\hline Mexican spiny pocket mouse & $\mathrm{S}$ & Low & $\mathrm{S}$ & Subterranean Ground & 1 \\
\hline \multicolumn{6}{|l|}{ Liomys irroratus } \\
\hline Small Japanese field mouse & S & Low & $N, S$ & Subterranean Ground & 1 \\
\hline \multicolumn{6}{|l|}{ Apodemus argenteus } \\
\hline Wood mouse & $\mathrm{S}$ & Low & $\mathrm{N}, \mathrm{S}$ & Subterranean Ground & 1 \\
\hline \multicolumn{6}{|l|}{ Apodemus sylvaticus } \\
\hline Yellow-necked mouse & S & Low & $N, S$ & Subterranean Ground & 1 \\
\hline \multicolumn{6}{|l|}{ Apodemus flavicollis } \\
\hline Fish crow & $\mathrm{S}$ & Mixed & $\mathrm{Mi}$ & Arboreal Surface & 2 \\
\hline \multicolumn{6}{|l|}{ Corvus ossifragus } \\
\hline African striped weasel & $\mathrm{S}$ & High & $\mathrm{Ca}, \mathrm{SM}$ & Arboreal Cavity & 3 \\
\hline
\end{tabular}


Table 1 A summary of caching behaviour of 203 vertebrates (adopted and updated from [164]). Caching behaviour includes duration of food storage, the perishability and type of food cached, and the location where food is stored are included within the table. In many cases, information on each of these three categories represent a 'best estimate', as much of the literature consists of anecdotal reports or natural history observations. All species were assigned a susceptibility score from 1-9 based on variation in two major aspects of caching behaviour: duration and food perishability (see text for details). Susceptibility to climate change was predicted to be lowest for short-term cachers of low perishable food item (1) and highest for long-term cachers of perishable food items (9). More research is needed to understand how location of storage may influence degradation of cached food and was, therefore, not included in the susceptibility score (Continued)

\begin{tabular}{|c|c|c|c|c|c|}
\hline \multicolumn{6}{|l|}{ Poecilogale albinucha } \\
\hline Barn owl & S & High & SM & Arboreal Cavity & 3 \\
\hline \multicolumn{6}{|l|}{ Tyto alba } \\
\hline Elf owl & S & High & । & Arboreal Cavity & 3 \\
\hline \multicolumn{6}{|l|}{ Micrathene whitneyi } \\
\hline Schreech owl & S & High & SM & Arboreal Cavity & 3 \\
\hline \multicolumn{6}{|l|}{ Otus asio } \\
\hline American kestrel & S & High & $\mathrm{Bi}, \mathrm{Re}, \mathrm{SM}$ & Arboreal Surface & 3 \\
\hline \multicolumn{6}{|l|}{ Falco sparverius } \\
\hline Barred owl & S & High & SM & Arboreal Surface & 3 \\
\hline \multicolumn{6}{|l|}{ Strix varia } \\
\hline Bat falcon & S & High & $\mathrm{Ba}, \mathrm{Bi}$ & Arboreal Surface & 3 \\
\hline \multicolumn{6}{|l|}{ Falco rufigulais } \\
\hline Boreal owl & S & High & SM & Arboreal Surface & 3 \\
\hline \multicolumn{6}{|l|}{ Aegolius funereus } \\
\hline Broad-winged hawk & S & High & $\mathrm{Bi}$ & Arboreal Surface & 3 \\
\hline \multicolumn{6}{|l|}{ Buteo platypterus } \\
\hline Buzzard & S & High & SM & Arboreal Surface & 3 \\
\hline \multicolumn{6}{|l|}{ Buteo buteo } \\
\hline Crowned eagle & S & High & MM & Arboreal Surface & 3 \\
\hline \multicolumn{6}{|c|}{ Stephanoaetus coronatus } \\
\hline Eagle owl & S & High & $\mathrm{Bi}$ & Arboreal Surface & 3 \\
\hline \multicolumn{6}{|l|}{ Bubo bubo } \\
\hline Eleonora's falcon & S & High & $\mathrm{Bi}$ & Arboreal Surface & 3 \\
\hline \multicolumn{6}{|l|}{ Falco eleonarae } \\
\hline Eurasian pygmy owl & S & High & $\mathrm{Bi}, \mathrm{SM}$ & Arboreal Surface & 3 \\
\hline \multicolumn{6}{|c|}{ Glaucidium passerinum } \\
\hline European kestrel & S & High & SM & Arboreal Surface & 3 \\
\hline \multicolumn{6}{|l|}{ Falco tinnunculus } \\
\hline Goshawk & S & High & $\mathrm{Bi}, \mathrm{SM}$ & Arboreal Surface & 3 \\
\hline \multicolumn{6}{|l|}{ Accipiter gentilis } \\
\hline Great horned owl & S & High & $\mathrm{SM}, \mathrm{MM}$ & Arboreal Surface & 3 \\
\hline \multicolumn{6}{|l|}{ Bubo virginianus } \\
\hline Leopard & S & High & LM, MM & Arboreal Surface & 3 \\
\hline \multicolumn{6}{|l|}{ Panthera pardus } \\
\hline Little owl & S & High & $\mathrm{Bi}, \mathrm{SM}$ & Arboreal Surface & 3 \\
\hline \multicolumn{6}{|l|}{ Athene noctua } \\
\hline Merlin & S & High & $\mathrm{Bi}, \mathrm{SM}$ & Arboreal Surface & 3 \\
\hline
\end{tabular}


Table 1 A summary of caching behaviour of 203 vertebrates (adopted and updated from [164]). Caching behaviour includes duration of food storage, the perishability and type of food cached, and the location where food is stored are included within the table. In many cases, information on each of these three categories represent a 'best estimate', as much of the literature consists of anecdotal reports or natural history observations. All species were assigned a susceptibility score from 1-9 based on variation in two major aspects of caching behaviour: duration and food perishability (see text for details). Susceptibility to climate change was predicted to be lowest for short-term cachers of low perishable food item (1) and highest for long-term cachers of perishable food items (9). More research is needed to understand how location of storage may influence degradation of cached food and was, therefore, not included in the susceptibility score (Continued)

\begin{tabular}{|c|c|c|c|c|c|}
\hline \multicolumn{6}{|l|}{ Falco columbarius } \\
\hline New Zealand falcon & $S$ & High & $\mathrm{Bi}$ & Arboreal Surface & 3 \\
\hline \multicolumn{6}{|l|}{ Falco novaehollandiae } \\
\hline Northern hawk owl & S & High & SM & Arboreal Surface & 3 \\
\hline \multicolumn{6}{|l|}{ Surnia ulula } \\
\hline Northern pygmy owl & S & High & SM & Arboreal Surface & 3 \\
\hline \multicolumn{6}{|l|}{ Glaucidium gnoma } \\
\hline Northern shrike & S & High & $\mathrm{Bi}, \mathrm{SM}$ & Arboreal Surface & 3 \\
\hline \multicolumn{6}{|l|}{ Lanius excubitor } \\
\hline Orange-breasted falcon & S & High & $\mathrm{Ba}$ & Arboreal Surface & 3 \\
\hline \multicolumn{6}{|l|}{ Falco deiroleucus } \\
\hline Peregrine falcon & S & High & $\mathrm{Bi}$ & Arboreal Surface & 3 \\
\hline \multicolumn{6}{|l|}{ Falco peregrinus } \\
\hline Prairie falcon & $\mathrm{S}$ & High & $\mathrm{Bi}$ & Arboreal Surface & 3 \\
\hline \multicolumn{6}{|l|}{ Falco mexicanus } \\
\hline Prevost's squirrel & $\mathrm{S}$ & High & $\mathrm{Fr}$ & Arboreal Surface & 3 \\
\hline \multicolumn{6}{|l|}{ Callosciurus prevosti } \\
\hline Saw-whet owl & S & High & SM & Arboreal Surface & 3 \\
\hline \multicolumn{6}{|l|}{ Aegolius acadicus } \\
\hline South island robin & S & High & । & Arboreal Surface & 3 \\
\hline \multicolumn{6}{|l|}{ Petroica australis } \\
\hline Sparrowhawk & S & High & $\mathrm{Bi}, \mathrm{SM}$ & Arboreal Surface & 3 \\
\hline \multicolumn{6}{|l|}{ Accipiter nisus } \\
\hline Tawny owl & $S$ & High & SM & Arboreal Surface & 3 \\
\hline \multicolumn{6}{|l|}{ Strix aluco } \\
\hline African wild dog & $S$ & High & LM, MM & Ground Surface & 3 \\
\hline \multicolumn{6}{|l|}{ Lycaon pictus } \\
\hline Barbados green monkey & S & High & $\mathrm{Fr}$ & Ground Surface & 3 \\
\hline \multicolumn{6}{|l|}{ Cercopithecus aethiops } \\
\hline Black bear & $\mathrm{S}$ & High & $\mathrm{Ca}, \mathrm{MM}, \mathrm{SM}$ & Ground Surface & 3 \\
\hline \multicolumn{6}{|l|}{ Ursus americanus } \\
\hline Black-backed jackal & $S$ & High & LM, SM, MM & Ground Surface & 3 \\
\hline \multicolumn{6}{|l|}{ Canis mesomelus } \\
\hline Black-billed magpie & $S$ & High & $\mathrm{Ca}, \mathrm{E}, \mathrm{N}, \mathrm{Mi}$ & Ground Surface & 3 \\
\hline \multicolumn{6}{|l|}{ Pica pica } \\
\hline Bobcat & S & High & $\mathrm{MM}, \mathrm{SM}$ & Ground Surface & 3 \\
\hline \multicolumn{6}{|l|}{ Lynx rufus } \\
\hline Brown bear & $\mathrm{S}$ & High & $\mathrm{Ca}, \mathrm{LM}, \mathrm{MM}$ & Ground Surface & 3 \\
\hline
\end{tabular}


Table 1 A summary of caching behaviour of 203 vertebrates (adopted and updated from [164]). Caching behaviour includes duration of food storage, the perishability and type of food cached, and the location where food is stored are included within the table. In many cases, information on each of these three categories represent a 'best estimate', as much of the literature consists of anecdotal reports or natural history observations. All species were assigned a susceptibility score from 1-9 based on variation in two major aspects of caching behaviour: duration and food perishability (see text for details). Susceptibility to climate change was predicted to be lowest for short-term cachers of low perishable food item (1) and highest for long-term cachers of perishable food items (9). More research is needed to understand how location of storage may influence degradation of cached food and was, therefore, not included in the susceptibility score (Continued)

\begin{tabular}{|c|c|c|c|c|c|}
\hline \multicolumn{6}{|l|}{ Ursus arctos } \\
\hline Canadian lynx & S & High & MM, SM & Ground Surface & 3 \\
\hline \multicolumn{6}{|l|}{ Lynx canadensis } \\
\hline Carrion crow & S & High & $\mathrm{Ca}$ & Ground Surface & 3 \\
\hline \multicolumn{6}{|l|}{ Corvus corone } \\
\hline Common crow & S & High & $\mathrm{A}, \mathrm{E}, \mathrm{Fi}, \mathrm{N}, \mathrm{SM}$ & Ground Surface & 3 \\
\hline \multicolumn{6}{|l|}{ Corvus brachyrhynchos } \\
\hline Common raven & S & High & $\mathrm{Ca}, \mathrm{E}, \mathrm{Mi}, \mathrm{SM}$ & Ground Surface & 3 \\
\hline \multicolumn{6}{|l|}{ Corvus corax } \\
\hline Coyote & S & High & LM, SM, MM & Ground Surface & 3 \\
\hline \multicolumn{6}{|l|}{ Canis latrans } \\
\hline European lynx & S & High & $\mathrm{MM}, \mathrm{SM}$ & Ground Surface & 3 \\
\hline \multicolumn{6}{|l|}{ Lynx lynx } \\
\hline Fennec fox & S & High & $\mathrm{E}, \mathrm{MM}, \mathrm{SM}$ & Ground Surface & 3 \\
\hline \multicolumn{6}{|l|}{ Vulpes zerda } \\
\hline Fisher & S & High & $\mathrm{Bi}, \mathrm{MM}, \mathrm{SM}$ & Ground Surface & 3 \\
\hline \multicolumn{6}{|l|}{ Martes pennanti } \\
\hline Golden jackal & $S$ & High & LM, SM, MM & Ground Surface & 3 \\
\hline \multicolumn{6}{|l|}{ Canis aureus } \\
\hline Lion & S & High & LM, MM & Ground Surface & 3 \\
\hline \multicolumn{6}{|l|}{ Panthera leo } \\
\hline MacGregor's bowerbird & S & High & $\mathrm{Fr}$ & Ground Surface & 3 \\
\hline \multicolumn{6}{|l|}{ Amblyornis macgregoriae } \\
\hline Mink & $S$ & High & $\mathrm{Bi}, \mathrm{MM}, \mathrm{SM}$ & Ground Surface & 3 \\
\hline \multicolumn{6}{|l|}{ Mustela vison } \\
\hline Mountain lion & S & High & LM, MM & Ground Surface & 3 \\
\hline \multicolumn{6}{|l|}{ Felis concolor } \\
\hline Northwestern crow & S & High & $\mathrm{Fi}, \mathrm{I}$ & Ground Surface & 3 \\
\hline \multicolumn{6}{|l|}{ Corvus caurinus } \\
\hline Pine marten & $\mathrm{S}$ & High & $\mathrm{Bi}, \mathrm{Ca}, \mathrm{MM}, \mathrm{SM}$ & Ground Surface & 3 \\
\hline \multicolumn{6}{|l|}{ Martes martes } \\
\hline Polar bear & $S$ & High & LM, MM & Ground Surface & 3 \\
\hline \multicolumn{6}{|l|}{ Ursus maritimus } \\
\hline Snowy owl & S & High & MM, SM & Ground Surface & 3 \\
\hline \multicolumn{6}{|l|}{ Nyctea scandiaca } \\
\hline Tiger & S & High & LM, MM & Ground Surface & 3 \\
\hline \multicolumn{6}{|l|}{ Panthera tigris } \\
\hline Wolf & $\mathrm{S}$ & High & LM, SM, MM & Ground Surface & 3 \\
\hline
\end{tabular}


Table 1 A summary of caching behaviour of 203 vertebrates (adopted and updated from [164]). Caching behaviour includes duration of food storage, the perishability and type of food cached, and the location where food is stored are included within the table. In many cases, information on each of these three categories represent a 'best estimate', as much of the literature consists of anecdotal reports or natural history observations. All species were assigned a susceptibility score from 1-9 based on variation in two major aspects of caching behaviour: duration and food perishability (see text for details). Susceptibility to climate change was predicted to be lowest for short-term cachers of low perishable food item (1) and highest for long-term cachers of perishable food items (9). More research is needed to understand how location of storage may influence degradation of cached food and was, therefore, not included in the susceptibility score (Continued)

\begin{tabular}{|c|c|c|c|c|c|}
\hline \multicolumn{6}{|l|}{ Canis lupus } \\
\hline Arctic shrew & $S$ & High & 1 & Subterranean Ground & 3 \\
\hline \multicolumn{6}{|l|}{ Sorex arcticus } \\
\hline Badger & S & High & $\mathrm{Ca}, \mathrm{MM}, \mathrm{SM}$ & Subterranean Ground & 3 \\
\hline \multicolumn{6}{|l|}{ Taxidea taxus } \\
\hline Burrowing owl & S & High & I, R & Subterranean Ground & 3 \\
\hline \multicolumn{6}{|l|}{ Athene cunicularia } \\
\hline European mole & S & High & I & Subterranean Ground & 3 \\
\hline \multicolumn{6}{|l|}{ Talpa europaea } \\
\hline Least weasel & S & High & SM & Subterranean Ground & 3 \\
\hline \multicolumn{6}{|l|}{ Mustela nivalis } \\
\hline Long-tailed weasel & S & High & SM & Subterranean Ground & 3 \\
\hline \multicolumn{6}{|l|}{ Mustela frenata } \\
\hline Masked shrew & S & High & I & Subterranean Ground & 3 \\
\hline \multicolumn{6}{|l|}{ Sorex cinereus } \\
\hline Mole-rat & S & High & $\mathrm{B}, \mathrm{V}$ & Subterranean Ground & 3 \\
\hline \multicolumn{6}{|l|}{ Spalax leucodon } \\
\hline Pygmy shrew & S & High & । & Subterranean Ground & 3 \\
\hline \multicolumn{6}{|l|}{ Microsorex hoyi } \\
\hline Short-tailed weasel & S & High & SM & Subterranean Ground & 3 \\
\hline \multicolumn{6}{|l|}{ Mustela erminea } \\
\hline Siberian mole & S & High & I & Subterranean Ground & 3 \\
\hline \multicolumn{6}{|l|}{ Talpa altaica } \\
\hline Water shrew & S & High & A, I, SM & Subterranean Ground & 3 \\
\hline \multicolumn{6}{|l|}{ Sorex palustris } \\
\hline Spotted hyena & S & High & LM, MM & Water & 3 \\
\hline \multicolumn{6}{|l|}{ Crocuta crocuta } \\
\hline Heather vole & Mixed & Low & W & Arboreal Surface & 4 \\
\hline \multicolumn{6}{|l|}{ Phenacomys intermedius } \\
\hline Eastern gray squirrel & Mixed & Low & $\mathrm{N}, \mathrm{S}, \mathrm{Mi}$ & Ground Surface & 4 \\
\hline \multicolumn{6}{|l|}{ Sciurus carolinensis } \\
\hline Eurasian red squirrel & Mixed & Low & Co, N, S & Ground Surface & 4 \\
\hline \multicolumn{6}{|l|}{ Scirus vulgaris } \\
\hline Fox squirrel & Mixed & Low & $\mathrm{N}, \mathrm{S}$ & Ground Surface & 4 \\
\hline \multicolumn{6}{|l|}{ Sciurus niger } \\
\hline Great basin pocket mouse & Mixed & Low & $S$ & Ground Surface & 4 \\
\hline \multicolumn{6}{|l|}{ Perognathus parvus } \\
\hline Hispid pocket mouse & Mixed & Low & $\mathrm{S}$ & Ground Surface & 4 \\
\hline
\end{tabular}


Table 1 A summary of caching behaviour of 203 vertebrates (adopted and updated from [164]). Caching behaviour includes duration of food storage, the perishability and type of food cached, and the location where food is stored are included within the table. In many cases, information on each of these three categories represent a 'best estimate', as much of the literature consists of anecdotal reports or natural history observations. All species were assigned a susceptibility score from 1-9 based on variation in two major aspects of caching behaviour: duration and food perishability (see text for details). Susceptibility to climate change was predicted to be lowest for short-term cachers of low perishable food item (1) and highest for long-term cachers of perishable food items (9). More research is needed to understand how location of storage may influence degradation of cached food and was, therefore, not included in the susceptibility score (Continued)

\begin{tabular}{|c|c|c|c|c|c|}
\hline \multicolumn{6}{|l|}{ Perognathus hispidus } \\
\hline Japanese squirrel & Mixed & Low & $\mathrm{Co}$ & Ground Surface & 4 \\
\hline \multicolumn{6}{|l|}{ Sciurus lis } \\
\hline Little pocket mouse & Mixed & Low & $\mathrm{S}$ & Ground Surface & 4 \\
\hline \multicolumn{6}{|l|}{ Perognathus longimembris } \\
\hline Long-tailed pocket mouse & Mixed & Low & $S$ & Ground Surface & 4 \\
\hline \multicolumn{6}{|l|}{ Perognathus formusus } \\
\hline Plains pocket mouse & Mixed & Low & S & Ground Surface & 4 \\
\hline \multicolumn{6}{|l|}{ Perognathus flavescens } \\
\hline Red-tailed squirrel & Mixed & Low & N & Ground Surface & 4 \\
\hline \multicolumn{6}{|l|}{ Sciurus granatensis } \\
\hline Rock pocket mouse & Mixed & Low & $\mathrm{S}$ & Ground Surface & 4 \\
\hline \multicolumn{6}{|l|}{ Perognathus intermedius } \\
\hline Tassel-eared squirrel & Mixed & Low & $\mathrm{N}, \mathrm{S}, \mathrm{Mu}$ & Ground Surface/Arboreal Surface & 4 \\
\hline \multicolumn{6}{|l|}{ Sciurus aberti } \\
\hline Alaska ground squirrel & Mixed & Low & $\mathrm{S}, \mathrm{V}$ & Subterranean Ground & 4 \\
\hline \multicolumn{6}{|l|}{ Spermophilus undulatus } \\
\hline Arctic ground squirrel & Mixed & Low & $S, V$ & Subterranean Ground & 4 \\
\hline \multicolumn{6}{|l|}{ Spermophilus parryii } \\
\hline Botta's pocket gopher & Mixed & Low & $\mathrm{S}$ & Subterranean Ground & 4 \\
\hline \multicolumn{6}{|l|}{ Thomomys bottae } \\
\hline Djungarian hamster & Mixed & Low & $\mathrm{N}, \mathrm{S}$ & Subterranean Ground & 4 \\
\hline \multicolumn{6}{|l|}{ Phodopus sungorus } \\
\hline Golden-mantled ground squirrel & Mixed & Low & $\mathrm{N}, \mathrm{S}$ & Subterranean Ground & 4 \\
\hline \multicolumn{6}{|l|}{ Spermophilus lateralis } \\
\hline Lesser bandicoot rat & Mixed & Low & $\mathrm{N}, \mathrm{S}, \mathrm{T}$ & Subterranean Ground & 4 \\
\hline \multicolumn{6}{|l|}{ Bandicota bengalensis } \\
\hline Mountain pocket gopher & Mixed & Low & V & Subterranean Ground & 4 \\
\hline \multicolumn{6}{|l|}{ Thomomys monticola } \\
\hline Muskrat & Mixed & Low & $B, R, T, V$ & Subterranean Ground & 4 \\
\hline \multicolumn{6}{|l|}{ Ondatra zibethicus } \\
\hline Northern pocket gopher & Mixed & Low & $\mathrm{R}, \mathrm{T}$ & Subterranean Ground & 4 \\
\hline \multicolumn{6}{|l|}{ Thomomys talpoides } \\
\hline Pouched mouse & Mixed & Low & $\mathrm{N}, \mathrm{S}$ & Subterranean Ground & 4 \\
\hline \multicolumn{6}{|l|}{ Saccostomus campestris } \\
\hline Richardson ground squirrel & Mixed & Low & $\mathrm{S}, \mathrm{V}$ & Subterranean Ground & 4 \\
\hline \multicolumn{6}{|l|}{ Spermophilus richardsonii } \\
\hline Rock squirrel & Mixed & Low & $\mathrm{N}, \mathrm{S}$ & Subterranean Ground & 4 \\
\hline
\end{tabular}


Table 1 A summary of caching behaviour of 203 vertebrates (adopted and updated from [164]). Caching behaviour includes duration of food storage, the perishability and type of food cached, and the location where food is stored are included within the table. In many cases, information on each of these three categories represent a 'best estimate', as much of the literature consists of anecdotal reports or natural history observations. All species were assigned a susceptibility score from 1-9 based on variation in two major aspects of caching behaviour: duration and food perishability (see text for details). Susceptibility to climate change was predicted to be lowest for short-term cachers of low perishable food item (1) and highest for long-term cachers of perishable food items (9). More research is needed to understand how location of storage may influence degradation of cached food and was, therefore, not included in the susceptibility score (Continued)

\begin{tabular}{|c|c|c|c|c|c|}
\hline \multicolumn{6}{|l|}{ Spermophilus variegatus } \\
\hline Syrian golden hamster & Mixed & Low & $S, R, T$ & Subterranean Ground & 4 \\
\hline \multicolumn{6}{|l|}{ Mesocricetus auratus } \\
\hline Thirteen-lined ground squirrel & Mixed & Low & $\mathrm{S}, \mathrm{V}$ & Subterranean Ground & 4 \\
\hline \multicolumn{6}{|l|}{ Spermophilus tridecemlineatus } \\
\hline Desert woodrat & Mixed & Mixed & V & Ground Surface & 5 \\
\hline \multicolumn{6}{|l|}{ Neotoma lepida } \\
\hline Eastern woodrat & Mixed & Mixed & V & Ground Surface & 5 \\
\hline \multicolumn{6}{|l|}{ Neotoma floridana } \\
\hline White-throated woodrat & Mixed & Mixed & V & Ground Surface & 5 \\
\hline \multicolumn{6}{|l|}{ Neotoma albigula } \\
\hline Bushy-tailed woodrat & Mixed & Mixed & V & Subterranean Ground & 5 \\
\hline \multicolumn{6}{|l|}{ Neotoma cinerea } \\
\hline Mexican woodrat & Mixed & Mixed & $\mathrm{S}, \mathrm{V}$ & Subterranean Ground & 5 \\
\hline \multicolumn{6}{|l|}{ Neotoma mexicana } \\
\hline Mountain beaver & Mixed & High & V & Subterranean Ground & 6 \\
\hline \multicolumn{6}{|l|}{ Aplodontia rufa } \\
\hline Arctic fox & Mixed & High & $\mathrm{E}, \mathrm{MM}, \mathrm{SM}$ & Ground Surface & 6 \\
\hline \multicolumn{6}{|l|}{ Alopex lagopus } \\
\hline Red fox & Mixed & High & $\mathrm{Bi}, \mathrm{E}, \mathrm{MM}, \mathrm{SM}$ & Ground Surface & 6 \\
\hline \multicolumn{6}{|l|}{ Vulpes vulpes } \\
\hline Short-tailed shrew & Mixed & High & $\mathrm{A}, \mathrm{Fi}, \mathrm{I}, \mathrm{SM}$ & Subterranean Ground & 6 \\
\hline \multicolumn{6}{|l|}{ Blarina brevicauda } \\
\hline Agouti & L & Low & $\mathrm{N}, \mathrm{S}$ & Ground Surface & 7 \\
\hline \multicolumn{6}{|l|}{ Dasyprocta punctata } \\
\hline Alipne chipmunk & L & Low & $\mathrm{N}, \mathrm{S}$ & Ground Surface & 7 \\
\hline \multicolumn{6}{|l|}{ Tamias alpinus } \\
\hline Blue jay & L & Low & $\mathrm{N}, \mathrm{Mi}, \mathrm{S}$ & Ground Surface & 7 \\
\hline \multicolumn{6}{|l|}{ Cyanocitta cristata } \\
\hline Clark's nutcracker & L & Low & $\mathrm{S}$ & Ground Surface & 7 \\
\hline \multicolumn{6}{|l|}{ Nucifraga columbiana } \\
\hline Cliff chipmunk & L & Low & $\mathrm{N}, \mathrm{S}$ & Ground Surface & 7 \\
\hline \multicolumn{6}{|l|}{ Tamias dorsalis } \\
\hline Eastern chipmunk & L & Low & I, N, S & Ground Surface & 7 \\
\hline \multicolumn{6}{|l|}{ Tamias striatus } \\
\hline Eurasian jay & L & Low & Co, N, S & Ground Surface & 7 \\
\hline \multicolumn{6}{|l|}{ Garrulus glandarius } \\
\hline Eurasian nutcracker & L & Low & $S$ & Ground Surface & 7 \\
\hline
\end{tabular}


Table 1 A summary of caching behaviour of 203 vertebrates (adopted and updated from [164]). Caching behaviour includes duration of food storage, the perishability and type of food cached, and the location where food is stored are included within the table. In many cases, information on each of these three categories represent a 'best estimate', as much of the literature consists of anecdotal reports or natural history observations. All species were assigned a susceptibility score from 1-9 based on variation in two major aspects of caching behaviour: duration and food perishability (see text for details). Susceptibility to climate change was predicted to be lowest for short-term cachers of low perishable food item (1) and highest for long-term cachers of perishable food items (9). More research is needed to understand how location of storage may influence degradation of cached food and was, therefore, not included in the susceptibility score (Continued)

\begin{tabular}{|c|c|c|c|c|c|}
\hline \multicolumn{6}{|l|}{ Nucifraga caryocatactes } \\
\hline Flat-headed vole & L & Low & V & Ground Surface & 7 \\
\hline \multicolumn{6}{|l|}{ Alticola strelzowi } \\
\hline Green achouti & L & Low & $\mathrm{N}, \mathrm{S}$ & Ground Surface & 7 \\
\hline \multicolumn{6}{|l|}{ Myoprocta acouchi } \\
\hline Least chipmunk & L & Low & $\mathrm{N}, \mathrm{S}$ & Ground Surface & 7 \\
\hline \multicolumn{6}{|l|}{ Tamias minimus } \\
\hline Lodgepole pine chipmunk & L & Low & $\mathrm{N}, \mathrm{S}$ & Ground Surface & 7 \\
\hline \multicolumn{6}{|l|}{ Tamias speciosus } \\
\hline North American pika & L & Low & V & Ground Surface & 7 \\
\hline \multicolumn{6}{|l|}{ Ochotona princeps } \\
\hline Pinyon jay & $L$ & Low & $\mathrm{N}, \mathrm{S}$ & Ground Surface & 7 \\
\hline \multicolumn{6}{|l|}{ Gymnorhinus cyanocephlus } \\
\hline Red achouchi & $L$ & Low & $\mathrm{N}, \mathrm{S}$ & Ground Surface & 7 \\
\hline \multicolumn{6}{|l|}{ Myoprocta exilis } \\
\hline Red-tailed chipmunk & L & Low & $\mathrm{N}, \mathrm{S}$ & Ground Surface & 7 \\
\hline \multicolumn{6}{|l|}{ Tamias ruficaudus } \\
\hline Western scrub jay & L & Low & $\mathrm{N}, \mathrm{S}$ & Ground Surface & 7 \\
\hline \multicolumn{6}{|l|}{ Aphelocoma coerulescens } \\
\hline Siberian chipmunk & L & Low & $\mathrm{N}, \mathrm{S}$ & Ground Surface & 7 \\
\hline \multicolumn{6}{|l|}{ Tamias sibiricus } \\
\hline Siberian pika & L & Low & V & Ground Surface & 7 \\
\hline \multicolumn{6}{|l|}{ Ochotona alpina } \\
\hline Steller's jay & L & Low & $\mathrm{N}, \mathrm{Mi}, \mathrm{S}$ & Ground Surface & 7 \\
\hline \multicolumn{6}{|l|}{ Cyanocitta stelleri } \\
\hline Yellow pine chipmunk & L & Low & $\mathrm{N}, \mathrm{S}$ & Ground Surface & 7 \\
\hline \multicolumn{6}{|l|}{ Tamias amoenus } \\
\hline Douglas'squirrel & L & Low & $\mathrm{Co}, \mathrm{Mu}, \mathrm{S}$ & Ground Surface/Arboreal Surface & 7 \\
\hline \multicolumn{6}{|l|}{ Tamiasciurus douglasii } \\
\hline Red squirrel & L & Low & $\mathrm{Co}, \mathrm{Mu}, \mathrm{S}$ & Ground Surface/Arboreal Surface & 7 \\
\hline \multicolumn{6}{|l|}{ Tamiascurus hudsonicus } \\
\hline African giant rat & L & Low & $\mathrm{N}, \mathrm{S}, \mathrm{T}$ & Subterranean Ground & 7 \\
\hline \multicolumn{6}{|l|}{ Cricetomys gambianus } \\
\hline Alaska vole & L & Low & V & Subterranean Ground & 7 \\
\hline \multicolumn{6}{|l|}{ Microtus miurus } \\
\hline Bank vole & L & High & V (Lichen) & Arboreal Cavity/Surface & 7 \\
\hline \multicolumn{6}{|l|}{ Clethrionomys glareolus } \\
\hline Banner-tailed kangaroo rat & $\mathrm{L}$ & Low & $\mathrm{S}$ & Subterranean Ground & 7 \\
\hline
\end{tabular}


Table 1 A summary of caching behaviour of 203 vertebrates (adopted and updated from [164]). Caching behaviour includes duration of food storage, the perishability and type of food cached, and the location where food is stored are included within the table. In many cases, information on each of these three categories represent a 'best estimate', as much of the literature consists of anecdotal reports or natural history observations. All species were assigned a susceptibility score from 1-9 based on variation in two major aspects of caching behaviour: duration and food perishability (see text for details). Susceptibility to climate change was predicted to be lowest for short-term cachers of low perishable food item (1) and highest for long-term cachers of perishable food items (9). More research is needed to understand how location of storage may influence degradation of cached food and was, therefore, not included in the susceptibility score (Continued)

\begin{tabular}{|c|c|c|c|c|c|}
\hline \multicolumn{6}{|l|}{ Dipodomys spectabilis } \\
\hline Black-bellied hamster & $\mathrm{L}$ & Low & $\mathrm{T}, \mathrm{V}$ & Subterranean Ground & 7 \\
\hline \multicolumn{6}{|l|}{ Cricetus cricetus } \\
\hline Brandt's vole & L & Low & $\mathrm{V}, \mathrm{WV}$ & Subterranean Ground & 7 \\
\hline \multicolumn{6}{|l|}{ Microtus brandti } \\
\hline California ground squirrel & L & Low & $\mathrm{S}, \mathrm{V}$ & Subterranean Ground & 7 \\
\hline \multicolumn{6}{|l|}{ Spermophilus beecheyi } \\
\hline Cape mole-rat & L & Low & $\mathrm{B}, \mathrm{R}, \mathrm{T}$ & Subterranean Ground & 7 \\
\hline \multicolumn{6}{|l|}{ Georychus capensis } \\
\hline Cape dune mole-rat & L & Low & $\mathrm{T}$ & Subterranean Ground & 7 \\
\hline \multicolumn{6}{|l|}{ Bathyergus suillus } \\
\hline Common mole-rat & $L$ & Low & $\mathrm{B}$ & Subterranean Ground & 7 \\
\hline \multicolumn{6}{|l|}{ Cryptomys hottentotus } \\
\hline Common vole & L & Low & B & Subterranean Ground & 7 \\
\hline \multicolumn{6}{|l|}{ Microtus arvalis } \\
\hline Coruro & L & Low & $\mathrm{B}, \mathrm{T}$ & Subterranean Ground & 7 \\
\hline \multicolumn{6}{|l|}{ Spalacopus cyanus } \\
\hline Daurian pika & L & Low & V & Subterranean Ground & 7 \\
\hline \multicolumn{6}{|l|}{ Ochotona daurica } \\
\hline Deer mouse & L & Low & $\mathrm{S}$ & Subterranean Ground & 7 \\
\hline \multicolumn{6}{|l|}{ Peromyscus maniculatus } \\
\hline Desert kangaroo rat & L & Low & $\mathrm{S}$ & Subterranean Ground & 7 \\
\hline \multicolumn{6}{|l|}{ Dipodomys deserti } \\
\hline Diurnal sand rat & L & Low & $\mathrm{S}, \mathrm{V}$ & Subterranean Ground & 7 \\
\hline \multicolumn{6}{|l|}{ Psammomys obesus } \\
\hline Edible doormouse & L & Low & $B, N, S$ & Subterranean Ground & 7 \\
\hline \multicolumn{6}{|l|}{ Myoxus glis } \\
\hline Forest dormouse & L & Low & $\mathrm{Co}, \mathrm{S}$ & Subterranean Ground & 7 \\
\hline \multicolumn{6}{|l|}{ Dryomys nitedula } \\
\hline Giant kangaroo rat & L & Low & $\mathrm{S}$ & Subterranean Ground & 7 \\
\hline \multicolumn{6}{|l|}{ Dipodomys ingens } \\
\hline Great basin kangaroo rat & L & Low & $\mathrm{S}$ & Subterranean Ground & 7 \\
\hline \multicolumn{6}{|l|}{ Dipodomys microps } \\
\hline Greater long-tailed hamster & L & Low & V & Subterranean Ground & 7 \\
\hline \multicolumn{6}{|l|}{ Cricetulus triton } \\
\hline Hazel doormouse & L & Low & Co, N, S & Subterranean Ground & 7 \\
\hline \multicolumn{6}{|l|}{ Muscardinus avellanarius } \\
\hline Heermann's kangaroo rat & L & Low & $\mathrm{S}$ & Subterranean Ground & 7 \\
\hline
\end{tabular}


Table 1 A summary of caching behaviour of 203 vertebrates (adopted and updated from [164]). Caching behaviour includes duration of food storage, the perishability and type of food cached, and the location where food is stored are included within the table. In many cases, information on each of these three categories represent a 'best estimate', as much of the literature consists of anecdotal reports or natural history observations. All species were assigned a susceptibility score from 1-9 based on variation in two major aspects of caching behaviour: duration and food perishability (see text for details). Susceptibility to climate change was predicted to be lowest for short-term cachers of low perishable food item (1) and highest for long-term cachers of perishable food items (9). More research is needed to understand how location of storage may influence degradation of cached food and was, therefore, not included in the susceptibility score (Continued)

\begin{tabular}{|c|c|c|c|c|c|}
\hline \multicolumn{6}{|l|}{ Dipodomys heermanni } \\
\hline Indian gerbil & $\mathrm{L}$ & Low & $S, V$ & Subterranean Ground & 7 \\
\hline \multicolumn{6}{|l|}{ Tatera indica } \\
\hline Meadow vole & L & Low & $\mathrm{R}$ & Subterranean Ground & 7 \\
\hline \multicolumn{6}{|l|}{ Microtus pennsylvanicus } \\
\hline Merriam's kangaroo rat & L & Low & $S$ & Subterranean Ground & 7 \\
\hline \multicolumn{6}{|l|}{ Dipodomys merriami } \\
\hline Mexican jay & L & Low & $\mathrm{N}, \mathrm{S}$ & Ground Surface & 7 \\
\hline \multicolumn{6}{|l|}{ Aphelocoma ultramarina } \\
\hline Mid-day gerbil & $L$ & Low & $\mathrm{N}, \mathrm{S}, \mathrm{T}, \mathrm{V}$ & Subterranean Ground & 7 \\
\hline \multicolumn{6}{|l|}{ Meriones meridianus } \\
\hline Mole-rat & $L$ & Low & $B, R, T, V$ & Subterranean Ground & 7 \\
\hline \multicolumn{6}{|l|}{ Spalax microphthalmus } \\
\hline Mole vole & L & Low & $\mathrm{B}, \mathrm{T}$ & Subterranean Ground & 7 \\
\hline \multicolumn{6}{|l|}{ Ellobius talpinus } \\
\hline Mountain pygmy possum & L & Low & $\mathrm{N}, \mathrm{S}$ & Subterranean Ground & 7 \\
\hline \multicolumn{6}{|l|}{ Burramys parvus } \\
\hline Namaqua gerbil & L & Low & $\mathrm{S}$ & Subterranean Ground & 7 \\
\hline \multicolumn{6}{|l|}{ Desmodillus auricularis } \\
\hline Nothern grasshopper mouse & L & Low & $\mathrm{S}$ & Subterranean Ground & 7 \\
\hline \multicolumn{6}{|l|}{ Onychomys leucogaster } \\
\hline Pale kangaroo mouse & L & Low & $S$ & Subterranean Ground & 7 \\
\hline \multicolumn{6}{|l|}{ Microdipodops pallidus } \\
\hline Pallas' pika & $\mathrm{L}$ & Low & V & Subterranean Ground & 7 \\
\hline \multicolumn{6}{|l|}{ Ochotona pallasi } \\
\hline Plains pocket gopher & $\mathrm{L}$ & Low & $\mathrm{B}, \mathrm{T}$ & Subterranean Ground & 7 \\
\hline \multicolumn{6}{|l|}{ Geomys bursarius } \\
\hline Prairie vole & $\mathrm{L}$ & Low & $S$ & Subterranean Ground & 7 \\
\hline \multicolumn{6}{|l|}{ Pitmys ochrogaster } \\
\hline Reddish-gray vole & $\mathrm{L}$ & Low & V & Subterranean Ground & 7 \\
\hline \multicolumn{6}{|l|}{ Clethrionomys rufocanus } \\
\hline Rook & L & Low & Co, N, S & Ground Surface & 7 \\
\hline \multicolumn{6}{|l|}{ Corvus frugilegus } \\
\hline Santa Cruz kangaroo rat & $\mathrm{L}$ & Low & $\mathrm{S}$ & Subterranean Ground & 7 \\
\hline \multicolumn{6}{|l|}{ Dipodomys venustus } \\
\hline Social vole & L & Low & $\mathrm{B}, \mathrm{R}$ & Subterranean Ground & 7 \\
\hline \multicolumn{6}{|l|}{ Microtus socialis } \\
\hline Southeastern pocket gopher & L & Low & $\mathrm{B}, \mathrm{T}$ & Subterranean Ground & 7 \\
\hline
\end{tabular}


Table 1 A summary of caching behaviour of 203 vertebrates (adopted and updated from [164]). Caching behaviour includes duration of food storage, the perishability and type of food cached, and the location where food is stored are included within the table. In many cases, information on each of these three categories represent a 'best estimate', as much of the literature consists of anecdotal reports or natural history observations. All species were assigned a susceptibility score from 1-9 based on variation in two major aspects of caching behaviour: duration and food perishability (see text for details). Susceptibility to climate change was predicted to be lowest for short-term cachers of low perishable food item (1) and highest for long-term cachers of perishable food items (9). More research is needed to understand how location of storage may influence degradation of cached food and was, therefore, not included in the susceptibility score (Continued)

\begin{tabular}{|c|c|c|c|c|c|}
\hline Spiny pocket mouse & L & Low & $\mathrm{Fr}, \mathrm{N}, \mathrm{S}$ & Subterranean Ground & 7 \\
\hline \multicolumn{6}{|l|}{ Heteromys desmarestianus } \\
\hline Taiga vole & L & Low & V & Subterranean Ground & 7 \\
\hline \multicolumn{6}{|l|}{ Microtus xanthognathus } \\
\hline Tamarisk gerbil & L & Low & $N, S, T$ & Subterranean Ground & 7 \\
\hline \multicolumn{6}{|l|}{ Meriones tamariscinus } \\
\hline Tree mouse & L & Low & $\mathrm{S}, \mathrm{N}$ & Subterranean Ground & 7 \\
\hline \multicolumn{6}{|l|}{ Beamys major } \\
\hline White-footed mouse & L & Low & $\mathrm{N}, \mathrm{S}$ & Subterranean Ground & 7 \\
\hline \multicolumn{6}{|l|}{ Peromyscus leucopus } \\
\hline American beaver & L & Mixed & $\mathrm{V}, \mathrm{WV}$ & Water & 8 \\
\hline \multicolumn{6}{|l|}{ Castor canadensis } \\
\hline Dusky-footed woodrat & L & Mixed & $N, S, V$ & Subterranean Ground & 8 \\
\hline \multicolumn{6}{|l|}{ Neotoma fuscipes } \\
\hline Eurasian beaver & L & Mixed & $\mathrm{V}, \mathrm{WV}$ & Water & 8 \\
\hline \multicolumn{6}{|l|}{ Castor fiber } \\
\hline Bull-headed shrike & L & High & $\mathrm{Bi}, \mathrm{I}, \mathrm{SM}$ & Arboreal Surface & 9 \\
\hline \multicolumn{6}{|l|}{ Lanius bucephalus } \\
\hline Gray jay & L & High & $\mathrm{Ca}, \mathrm{Fr}, \mathrm{I}, \mathrm{Mi}$ & Arboreal Surface & 9 \\
\hline \multicolumn{6}{|l|}{ Perisoreus canadensis } \\
\hline Siberian jay & L & High & $\mathrm{Ca}, \mathrm{Fr}, \mathrm{I}$ & Arboreal Surface & 9 \\
\hline \multicolumn{6}{|l|}{ Perisoreus infaustus } \\
\hline Wolverine & L & High & LM, MM & Ground Surface & 9 \\
\hline Gulo gulo & & & & & \\
\hline
\end{tabular}

Legend: $A$ amphibian, $B$ bulb, $B a$ bat, $B i$ bird, Ca carrion, Co cone, $E$ eggs, Fi fish, Fr fruit, $I$ invertebrates, $L M$ large mammal, $N$ nuts, $M i$ miscellaneous human foods, $M M$ medium mammal, $M u$ mushroom, $R$ roots, Re reptiles, $S$ seeds, $S M$ small mammal, $T$ tuber, $V$ vegetation, $W V$ woody vegetation. Note that mammal classifications are based off of Vander Wall [164]

herbivores, such as Collared Pikas (Ochotona collaris), and Agoutis (Dasyprocta punctata) have been documented to store both non-perishable nuts, and seeds, and perishable fruit $[38,71,92,134,165]$.

Below, we discuss how the field of food science can be used to develop an understanding of conditions that maintain and degrade perishable food items. Although there are few studies in food science that examine the influence of the environment on seeds and nuts, we also discuss how the field of plant biology may offer some insight into how variation in climate could influence germination rates and reduce the functional quality of stored seeds.

\section{Location of food storage}

With the exception of Beavers (Castor canadensis and Castor fiber) and Spotted Hyenas (Crocuta crocuta) which cache food in water, caching species store food in two types of locations: in the ground or in trees [164]. When food is stored below ground it will tend to be more buffered from environmental conditions compared to food that is stored above ground. For example, when air temperatures are below zero, temperatures remained above freezing in underground burrows of Alpine Marmots (Marmota marmot; [5]). When food is stored during the temperate zone winter, snow accumulation of $30-40 \mathrm{~cm}$ can decouple soil and air temperature [156]. 


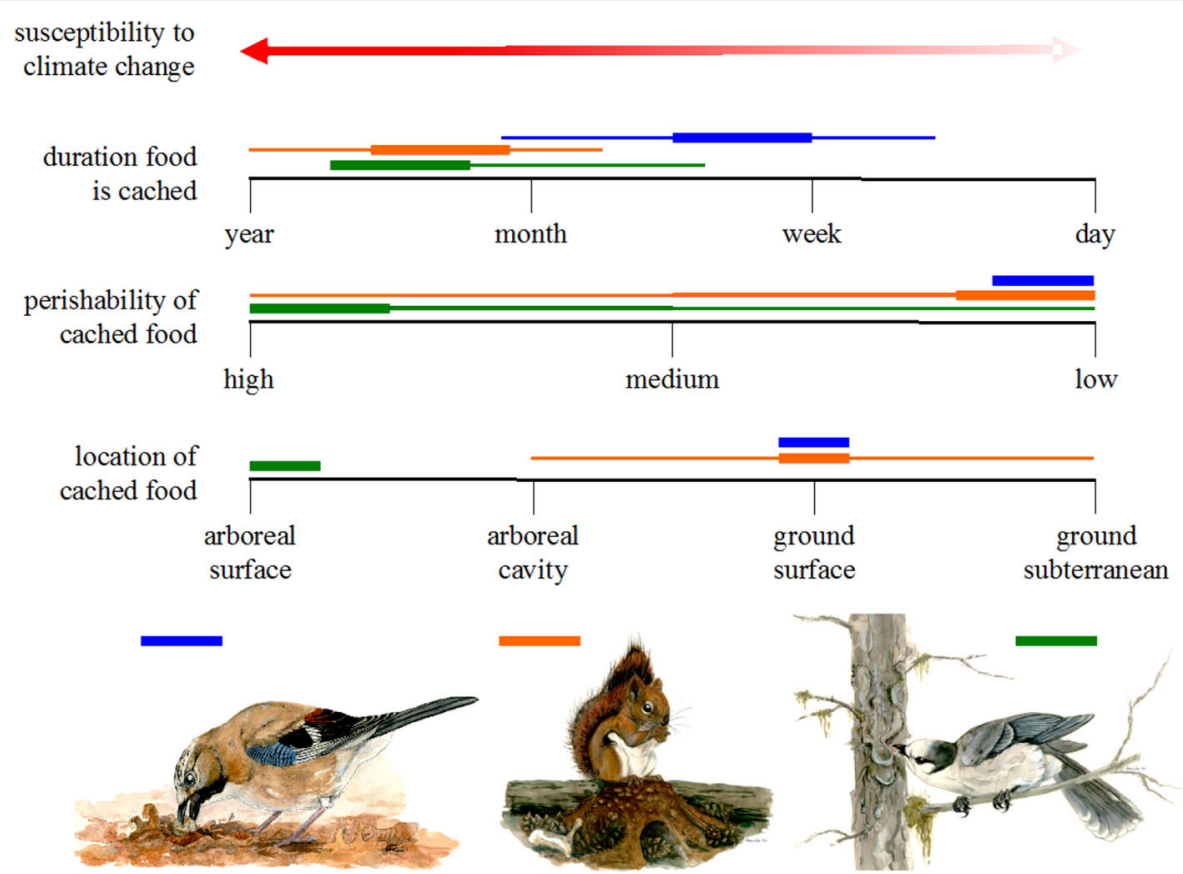

Fig. 1 Three axes hypothesized to influence the susceptibility of caching species to climate change with examples from species. Duration is the length of time that a food item is stored, perishability is how susceptible a food item is to microbial degradation and location is where food is stored. The latter falls under four categories ranging from high (arboreal) to low (subterranean) susceptibility. Each line represents the variation within a species on a particular axes with the thickness of a line representing the primary place in which a species falls along a given axis. For example, Gray Jays store perishable food but this storage can range from weeks to months. Green represents Gray Jays, blue represents Eurasian Jays and orange represents Red Squirrels. Illustrations by A. Gubbe

In contrast, when food is cached above ground, it is more likely to be directly exposed to environmental conditions. For example, White-breasted Nuthatches (Sitta carolinensis) store food caches in exposed bark crevices on the trunk and limbs of trees [75, 116, 179], while Jays of the genus Perisoreus cache food under pieces of bark or lichen on branches [36, 154]. Other species, such as Leopards, leave food items conspicuously on branches where it is difficult for competitors, such as Lions and Spotted Hyenas, to access them ([35], Eltringham SK. The ecology and conservation of large African mammals. [39]). In contrast, some species, such as Boreal Owls (Aegolius funereus), store food in tree cavities [79], which likely offers greater protection from environmental conditions compared to food stored on the exterior of a tree.

Regardless of whether species store above or below ground, some species may also exploit different microhabitats. For example, Banner-tailed Kangaroo Rats (Diopdomys spectabilis) store food in multiple chambers within their complex subterranean burrows [134]. Storage chambers can vary in both humidity and temperature, resulting in differential microbial activity between chambers [133]. Banner-tailed Kangaroo Rats prefer seeds with intermediate levels of mould and, therefore, exploit differences in microhabitats by moving seeds with high mould levels to low humidity chambers, and seeds with low mould levels to high humidity chambers [133]. Differences in cache placement (subterranean burrows, ground surface, tree cavities and tree surfaces) mean that food items will be exposed to different climatic conditions, which could lead to variation in the degree of degradation. It is clear that additional research is required to better understand the extent to which exposure to environmental conditions differ between caching locations.

\section{Summary of trends and susceptibility estimates}

We compiled a list of 203 caching species for which there is information on both the duration of storage and perishability of cached food (Table 1). From this information, we developed a susceptibility score for each species using a hierarchical classification system. Species were first divided into three categories based on the duration of food storage (short, mixed or long) and then, within each of these three categories, further divided into three groups according to the degree of perishability of cached food (low, mixed or high). We considered what are typically called 'non-perishable' food (nuts, seeds) as low perishability because climate may influence germination rates (see food science and plant biology 
discussions below). For both storage duration and perishability, species were defined as 'mixed' when there was evidence in the literature for both 'high' and 'low' storage duration or perishability. This classification created nine possible categories, [1] short-term, lowperishability, [2] short-term, mixed-perishability, [3] short-term, high-perishability, [4] mixed duration, lowperishability, [5] mixed duration, mixed-perishability, [6] mixed duration, high-perishability, [7] long-term, lowperishability, [8] long-term, mixed perishability, and [9] long-term, high-perishability food. High scores represent the greatest predicted susceptibility to climate change. Storage location was not used to determine susceptibility scores because there is not yet enough empirical evidence about how food cached at these different locations (e.g. subterranean vs. arboreal surface) is influenced by environmental conditions.

Because of the short-term nature of their caching behaviour, the largest proportion of caching species (44\%) were assigned to the three lowest susceptibility scores (1-3). Of the $38 \%$ of species in the three highest susceptibility categories (score of 7-9), 91\% relied on low-perishability food (score of 7). Seven percent (7\%) stored both high- and low-perishability food and only 2\% (four species: the Bull-headed Shrike Lanius busephalus, Gray Jay, Siberian Jay Perisoreus infaustus and Wolverine Gulo gulo) were both long-term cachers and relied exclusively on high-perishability food (susceptibility score of 9). Population declines at the southern edge of ranges have already been documented for both Gray Jays [171] and Wolverines [6] and studies on both of these species also suggest that climate change could be contributing to population declines [62, 139, 171].

A smaller proportion of species $(18 \%)$ were classified as mixed duration cachers (e.g. Artic Foxes, Alopex lagopus and Red Foxes, Vulpes vulpes, susceptibility scores of 6). The reason why some species are classified as mixed duration cachers may partly reflect geographic differences in caching behaviour within a species [43, 90, 168]. However, most species or populations likely cache a variety of food items with different degrees of perishability (Fig. 1). In such cases, food that is more perishable may also be of higher nutritional value (e.g. meat), which implies that species in this category may be more susceptible to climate change than we have estimated here. Nevertheless, it is clear that, for many species, more information is required to quantify how long food is stored, as well as the proportion of specific food items that are stored and their degree of perishability. Such information will improve our estimates of susceptibility and will, therefore, be important for understanding how climate change could influence their abundance.

\section{Integrating concepts from food science to understand the susceptibility of perishable food to climate change}

Understanding how environmental conditions influence food quality is a major focus of the field of food science $[107,122]$. A number of conditions have been identified that can degrade or preserve a wide range of perishable food consumed by humans [16, 86, 97, 107]. At the most general level, food can be degraded in three ways. First, even in the complete absence of bacteria or fungi, food may lose nutritional quality through a breakdown in structure due to a number of physical and chemical processes $[10,16]$. Second, microbial proliferation in food leads to losses of the nutrients and energy originally available to non-microbial competitors (e.g., humans) as these resources are diverted into the growth of indigestible bacteria [51, 59, 60]. Third, even when food still contains energy and nutrients potentially useful to non-microbes, these resources may be rendered inaccessible as many bacteria (e.g., Staphylococcus aureus and Clostridium botulinum) produce toxins or noxious substances that induce vomiting, diarrhea or otherwise render food inedible for humans [51, 52]. Although spoilage is fundamentally different from the physical or microbial degradation of a food item, in nature the three processes can be considered tightly linked. For example, the physical degradation of a food item (e.g. through freeze-thaw cycles) may accelerate microbial proliferation, which could then enhance the production of toxins that render food inedible. Thus, these processes will likely have to be considered together as they will be difficult to separate in the wild. Here, we focus on four classes of environmental factors that are recognized to influence perishable food stored by humans and that we believe are relevant to natural systems.

\section{Temperature}

Temperature has long been the subject of study in the field of food science because it influences microbial growth, with different temperature thresholds responsible for facilitating or inhibiting microbial growth depending on the food item and species of bacteria [107]. Generally, warm temperatures act to facilitate microbial growth, whereas cold temperatures inhibit growth [23]. Humans have manipulated temperature for centuries to extend the duration for which food can be stored $[107,151]$. Lowering temperature is extremely effective because it acts to retard microbial growth across a range of food groups, such as meat, fruit and vegetative matter [22, 23, 129].

Many studies have investigated the relationship between temperature and microbial growth (e.g. [9, 20, 21, 49, 184]). Zwietering et al. [184] focused on modelling 
the relationship between temperature and bacterial growth rate. Simple models, including a linear relationship between temperature and growth rate and constant growth at all temperatures were not supported. Instead, the best fitting models were derived from a square root model originally proposed by Ratkowsky et al. [131]. Part of the reason why simpler models show a lack of fit is that asymptotes exist to bacterial growth, particularly at extreme temperatures [184]. These studies also highlight the importance of both bacterial species and the food substrate in determining rates of bacterial growth at different temperatures. For example, Bovill et al. [21] demonstrated that the proliferation of Listeria monocytogenes and Salmonella spp. at the same temperature depended on substrate (milk vs. broth vs. meat) and bacteria species. This dependence is likely the result of competition between the existing bacterial flora of a food item and novel bacteria [119]. In natural systems, local bacterial communities could prevent additional harmful bacteria from colonizing a food item, preventing food spoilage due to the accumulation of noxious substances.

The majority of studies on the effects of climate change in wild animal populations have focused on the effect of temperature (e.g. [40, 77]) and temperature is certainly the most common environmental predictor variable used in studies of caching species as well (e.g. $[106,171])$. The advantage is that temperature is the most commonly recorded long-term environmental variable and, where it is not recorded, several models are available to estimate past temperature values on either a monthly or annual basis [94]. Using mean temperature values over a specific time period is clearly convenient, but we argue, perhaps not always the only ecologically relevant factor for caching animals because it may not capture other environmental conditions that are associated with different mechanisms known to influence the quality of stored food.

\section{Deep freezing}

It may be useful to separate deep freeze from the general effects of temperature because it represents a specific threshold below which microbial growth is halted rather than simply retarded. The specific temperature associated with a deep freeze event will depend on the microbe in question as cold tolerance varies across species [69]. As microbial activity is a major cause of food spoilage, stopping this process to preserve food over long time periods has been a major focus in the field of food science $[17,41]$. One study suggested that temperatures as low as $-55^{\circ} \mathrm{C}$ represent ideal storage conditions for meat [55] because enzymatic reactions and oxidative rancidity cease completely, removing most of the key processes that would degrade food quality [182]. However, temperatures do not necessarily need to be this extreme to halt microbial activity over time. A number of studies have found that bacteria and fungi on frozen food generally cease growth at $-8^{\circ} \mathrm{C}$ [45] and other research has provided evidence that the growth of some microbes halts at around $-12^{\circ} \mathrm{C}[42,98]$.

Perhaps more importantly, deep freeze events can also cause cell death or injury to microbial cells [113]. If microbes are killed when exposed to deep freeze temperatures, it means that subsequent degradation will occur at a slower rate, as fewer bacteria will be present to deplete nutritional resources or render a food item inedible through spoilage when temperatures increase [91, 174]. However, if not all bacteria are killed, deep freeze events can also act as a selection agent to promote cold tolerance [174]. Many bacterial species can also enter a 'viable but nonculturable' (VBNC) state, characterized by a large reduction in metabolic activity in response to extreme temperatures and other environmental stressors $[74,113]$. Once in the VBNC state, bacteria remain dormant until conditions facilitate resuscitation. Some studies suggest that an increase in temperature can result in resuscitation of cells in the VBNC state [114, 115, 177]. This highlights the importance of understanding the relationship between temperature and the activity of microbes present on cached food. In particular, understanding relevant thresholds that inhibit bacterial growth or kill bacteria will be key to interpreting the influence of climatic variables on food quality over time.

For caching species, deep freeze events could play a significant role in preserving the quality of stored food, in particular for species that store food for use over the winter. For example, in a study of the caloric content of simulated Gray Jay caches at three different latitudes in Ontario, Canada, Scheley et al. [139] found that the mass loss and caloric content of cached food (mealworms and raisins) was similar between low- and midlatitude sites even though these sites differed in mean fall temperatures. However, food caches from the highlatitude site lost less weight and caloric content, leading Scheley et al. [139] to speculate that 'deep freeze' events may be driving this pattern as the high-latitude site was the only site that reached an average of $-16^{\circ} \mathrm{C}$ during the winter. In Finland, bacterial activity was halted in two different decomposer communities in soil at $-16^{\circ} \mathrm{C}$, suggesting that this may be an important threshold in natural systems [157]. Despite these indirect lines of evidence, there have been no studies that have experimentally examined whether deep freeze events contribute to the preservation of perishable cached food of a wild animal.

\section{Humidity}

Another influence on microbial growth is the amount of water in the surrounding environment $[1,7]$. Humidity, 
a measure of the moisture content of air, influences the transfer of moisture between the air and surface of adjacent substrates [76]. It is well known that increases in moisture around a food item leads to increased microbial growth and proliferation [161]. For example, lower environmental moisture content has been found to decrease microbial growth on rice and flour and, consequently, increase the length of time these food items can be stored $[1,47]$.

Since high ambient humidity facilitates microbial degradation and spoilage [1], pronounced seasonal fluctuations in rainfall and ambient humidity may mean that during large portions of the year it is likely not profitable to store perishable food, particularly at low latitudes. In contrast, high-latitude ecosystems have lower ambient humidity levels, punctuated by increases in moisture in the form of rain and snow. This reduction in humidity favours long-term storage and could also allow species to store at multiple time points throughout the year.

At high latitudes, humidity is likely to influence food caches primarily in the spring, summer, and fall because warm air can hold a larger quality of water vapour [162] and this means that more water is available to microbes [82]. Conversely, in winter, much of the moisture is present as a solid (i.e. ice), which means that it cannot be easily accessed by microbes, thus inhibiting growth.

\section{Freeze-thaw events}

Freeze-thaw events cause phase changes of bound water within a cell (e.g. ice crystal formation) and the associated rapid expansion and contraction of water can result in damage to cellular structures $[10,86,87,170]$. As a result of the degradation of cellular structures, freeze-thaw events can affect multiple aspects of food quality and several studies in applied food science have been conducted to understand the mechanisms behind these processes.

Microstructure is one major component of food quality that is heavily influenced by freeze-thaw events $[3,10]$. The denaturation of proteins, particularly within meat and fish products, has been linked to changes to the microstructure of a food item $[3,16]$. Associated with these changes in microstructure is a process known as 'drip loss' [182]. As a food item undergoes a freezethaw cycle, damaged cells leak an exudate containing soluble nutrients, vitamins, minerals and protein $[10,86]$. In addition to siphoning nutritional content away from the cell, this exudate also produce favourable conditions for microbial growth by increasing nutrient and moisture available around a food item [86].

The number of freeze-thaw events that a food item experiences can also influence the amount of damage that is caused. Multiple freeze-thaw events can have strong additive effects that can cause food to degrade more rapidly $[16,68,128,148,150,180,181]$. Srinivasan et al.
[150] documented an increase in mechanical damage sustained by freshwater prawns (Macrobrachium rosenbergii) exposed to repeated freeze-thaw events and this damage was caused by repeated melting and reformation of ice-crystals within a cell. Boonsumrej et al. [16] found similar mechanical damage to Tiger Shrimp (Penaeus monodon) characterized by torn muscle fibres, an increase in distance between adjacent muscle fibres, and a breakdown of the subcuticular membrane surrounding muscle fibres. This mechanical damage was associated with increased thawing loss (the weight lost by a sample when comparing frozen and thawed weight), a decrease in protein concentrations and an increase in thiobarbituric acid, a compound associated with food decomposition [16].

In the field of food science, studies have typically examined the effect of up to five consecutive freeze-thaw events on food quality (e.g. [68, 148, 180, 181]). However, one study demonstrated that meat might continue to degrade after 15 freeze-thaw cycles [128]. Further studies are needed to determine if various food types have different threshold numbers of freeze-thaw cycles beyond which no further damage can be inflicted. Such studies would be important in order to determine if possible increases in the number of freeze-thaw events resulting from long-term changes in climate could decrease survival of food caches in the wild.

\section{Integrating concepts from plant biology to understand the susceptibility of non-perishable food to climate change}

Although cached seeds are typically considered nonperishable, seed become inedible when they germinate and therefore, may also be influenced by climate change. The field of plant biology has identified a number of regulatory processes and environmental conditions that influence the likelihood of germination [11]. For example, dormancy, the failure of a seed to germinate when conditions are otherwise favourable to promote germination [34, 46], ensures that seeds will only germinate when conditions are favourable for growth $[34,169]$ and is greatly influenced by a number of environmental variables including temperature and moisture.

\section{Temperature}

The effect of temperature is generally related to the life history of the plant species or taxa [11]. For example, winter annuals require periods of warm temperatures preceding cold temperatures in order for their seeds to germinate [12], whereas summer annuals require periods of cold weather followed by warm temperatures in order to germinate. Patterns of temperature fluctuations can also be important to stimulate germination, with many 
species responding favourably to alternating temperatures [84, 109, 144, 149].

Freeze-thaw cycles may also influence the germination of seeds that are stored by many food-caching species. Soil temperature regimes, which can be influenced by freeze-thaw events, have a strong bearing on the occurrence and timing of germination [126, 127], however this relationship is highly variable between plant species [14]. Several studies indicate that exposure to low soil temperatures is necessary for germination to occur $[78,143]$ and other studies have shown that freeze-thaw cycles can lead to scarification, a necessary precondition for germination in some plant species [183]. Van Assche et al. [163] proposed that freeze-thaw cycles could interact with cold winter temperatures in a two-step process to promote germination. First, low winter temperatures make seeds sensitive to freeze-thaw cycles. Second, freeze-thaw cycles cause seeds to become water permeable, facilitating germination. For such species, warming winter temperatures could lead, in the short term, to prolonged availability of food caches, as seeds would fail to germinate and, in the long term, to eventual local extinction of the trees/plants producing the seeds favoured by food-caching species. On the other hand, for plant species whose seeds germinate independently of exposure to cold temperatures or freeze-thaw cycles, warmer and shorter winters could shorten the availability of seeds to any animals that cached them [93].

\section{Moisture}

In general, some moisture is required to facilitate germination but the optimum water content varies across species [111]. Soaking seeds is a common commercial method used to "prime" seeds for germination, leading to a higher percentage of germination for many species $[2,118]$. Similar to temperature, variability in moisture is necessary for many species to promote germination. However, for some species, variability in moisture levels can result in a decreased germination rates or have no effect at all $[13,89,159]$. There may also be strong interactive effects between moisture and temperature. For example, in seeds without sufficient water content, dormancy will not be broken by temperature alone [11].

\section{Linking food-degrading environmental conditions with climate change}

The environmental variables outlined above are particularly relevant to natural systems as they are rapidly shifting due to climate change [63]. Already mean temperatures across the globe have increased [63, 173], precipitation patterns have shifted resulting in altered moisture regimes $[147,152]$ and an increase in unpredictable weather patterns, such as mid-winter thaws and late frosts, have been documented [63, 173]. The shift in these environmental variables suggests that the relationship between caching species and their environment is changing and potentially altering the benefits of caching food. However, it is important to note that not all of these environmental variables will necessarily shift in the same way or with similar magnitudes.

\section{Extracting climatic variables from historical weather data}

Historical weather records are valuable for quantifying how climatic variables may influence long-term changes in abundance of caching species. Even when data are sparse, minimum, maximum and mean temperatures can be used to estimate other climatic variables, such as freeze-thaw events and the duration of deep freeze events. Natural history characteristics, such as when a species begins caching food and when it retrieves cached food, should be used to determine relevant time points to extract data from historical records.

\section{Freeze-thaw events}

Extracting information on freeze-thaw events from historical records requires knowledge of food-specific initial freezing point [103]. Initial freezing points are directly related to the concentration of solutes in a food item and its water content [130] and are known for a variety of food items. Many of these estimates could be used as surrogates for food items cached by wild species. Missing from the existing literature, however, are estimates of arthropod initial freezing points, which are relevant to a number of food-caching species that regularly store this taxa. Once initial freezing points have been determined by experiments or estimated from the literature, the numbers of freeze-thaw events can then be extracted from historical weather records by determining the point when the temperature drops below and then rises above the initial freezing point.

\section{Deep-freeze Events}

Although deep-freeze events can be easily extracted from historical temperature records, the use of minimum, mean or maximum daily temperatures has an important bearing on how deep-freeze events are interpreted. For example, extracting deep-freeze events based on minimum daily temperature implies, in most cases, that temperatures will drop below the deep-freeze threshold for only part of the day. Alternatively, using maximum daily temperatures implies that temperatures will remain below the given deep freeze threshold for the entire day. Maximum daily temperatures allow for the estimation of deep-freeze days, which is the number of complete days that microbial activity is inhibited. However, without hourly weather records it is difficult to estimate exactly how long deep freeze events would inhibit microbial growth. To better 
predict deep-freeze thresholds, it is also important to understand the species or groups of microbes that are present in a food caching system. An understanding of the microbial diversity present on a food item could provide better estimates on the temperature at which microbial activity is halted, rather than relying on estimates obtained from the literature.

\section{Humidity}

Estimates of humidity from historical records can be difficult to obtain, as many weather stations have not recorded daily humidity [44]. In spite of this, proxies can be used to provide estimates of humidity or moisture levels in the environment. Rainfall and snowfall are climatic variables that are commonly found in historical weather records and can be used to provide a crude estimate of moisture in the environment. However, predictive models based on precipitation in combination with minimum daily temperature have been shown to provide better estimates of humidity in both North America and Europe. However, in more arid environments, such as parts of Africa, this relationship does not seem to be robust [44] and it is necessary to use more complex models [76].

\section{Characteristics of caching species that could mitigate the impact of climatic change \\ Behavioural strategies}

Caching species have developed a number of behavioural strategies that retard cache degradation $[37,67,95,135$, 158] and, therefore, may mitigate the effects of changing climate. These strategies include handling techniques, exploitation of chemical properties in the environment, and exploitation of certain climatic factors to decrease food perishability.

Several species have been documented to use specific handling techniques that lessen degradation of a cached food item. For example, incapacitating, rather than killing prey at the time of capture can serve to inhibit spoilage or reduce the rate of degradation. Burrowing Owls (Athene cunicularis) have been shown to incapacitate long-horned beetles to facilitate storage [135], while Elf Owls (Micrathene whitneyi) have been observed to damage the thorax and remove the legs from live sphinx months [88]. Other animals, such as the Short-tailed Shrew (Blarina brevicauda), produce toxins in their saliva that immobilize prey by rendering them comatose [95]. Once in this state, prey can remain alive for several days after capture.

Some species may exploit antimicrobial compounds in the environment to preserve cached food. Elgmork [37] suggested that Brown Bears (Ursus arctos) cover carcasses with Sphagnum moss to aid in long-term preservation because Sphagnum is known to contain phenolic compounds that have antimicrobial properties. Arboreal caching species may exploit similar antimicrobial compounds of coniferous trees (e.g. spruce Picea spp.), which have been proposed to preserve cached food better than deciduous trees. In Gray Jays, evidence suggests that territory quality at the southern edge of their range is related to the percentage of conifers on their territories $[110,155]$, which appears to be partly due to the superior ability of conifers to preserve food [155]. Willow Tits (Parus montanus) have also been observed to preferentially cache food on conifers rather than deciduous trees [81], which could also be related to the antimicrobial properties of conifers.

A third method that caching species use to retard microbial degradation over time is to exploit microhabitats and climatic conditions. Tigers have been documented to cache prey in areas with increased brush and cover prey items in debris. Schaller [138] suggested that these this was done to lower the temperature experienced by a food item to reduce microbial activity. Many rodents dry grass and berries [145] and Red Squirrels hang mushrooms in trees [56]. This drying process is analogous to freeze-drying human food, which increases the length of time food can be stored [132, 137]. Cones, however, are susceptible to disintegration through drying which may explain why squirrels place cones in terrestrial middens, where moisture levels are higher than above the ground or snow [158].

Species that have developed behavioural strategies to mitigate the influence of exposure to the environment may be less susceptible to the effects of climate change. For example, dried food will likely be less influenced by freeze-thaw cycles, as damage due to the phase change of water will be reduced. However, it is important to note that examples of species exploiting the environment to enhance preservation are largely anecdotal and, therefore, require more rigorous study.

\section{Physiological adaptations}

To our knowledge, no studies have explicitly investigated potential physiological adaptations of caching species to cope with microbial proliferation on cached food items but studies of scavengers could help to inform future research on this subject. Recently, Roggenbuck et al. [136] characterized a variety of adaptations in the digestive tracts of two New World vultures, Coragyps aratus and Cathartes aura. Both species were found to have low $\mathrm{pH}$ in their digestive systems that destroyed most bacterial species before they could reach the hindgut. Additionally, the intestinal microbiome of both species had a high prevalence of both Clostridia and Fusobacteria [136], which are commonly found on carrion. Their abundance in the hindgut likely benefits vultures by further breaking down carrion, allowing for the more 
complete digestion of food [136]. Both Clostridia and Fusobacteria have been demonstrated to cause a variety of negative effects in both wildlife and humans [53, 61, 80]. Their presence in vulture intestinal tracts suggests that they tolerate bacterial toxins, a finding also documented in other scavenging birds [112]. It is possible that food-caching species also possess similar physiological adaptations to eliminate harmful microbes that colonize stored food.

Specialized gut microbiomes could also allow caching species to cope with microbial colonization of a cache or digest rotting food. These adaptations could be particularly important for species that cache perishable food items, which are more likely to be colonized by bacteria. Such adaptations could buffer the impact of increased degradation arising from climate change. Investigations of gut microbiomes have been undertaken for a diverse range of species, including amphibians, reptiles and mammals (e.g. [72, 85, 101]), including one caching species (Red Squirrel; [153]).

\section{Conclusions}

We outline a novel approach to address how changes in the environment may influence food-caching species by synthesizing information from the fields of food science and plant germination ecology and then classifying the vulnerability of species based on caching behaviour. As caching species rely on stored food for survival during periods of limited food availability and, in some cases, for reproduction, factors that influence food quality could have major downstream effects on fitness and population dynamics.

Studies on both the Gray Jays [171] and Wolverines $[6,62]$ highlight how climate could be influencing population abundance but detailed demographic studies on caching species remain limited. It will also be important to consider what cached food is being used for during periods of low resource availability. For example, both Gray Jays and Wolverines use cached food not just for survival but also for reproduction [28, 154], meaning that multiple demographic vital rates may be linked to changes in cached food quality over time. Identifying the vital rates driving population dynamics will help to identify how the downstream effects of climate change on cached food quality may influence population growth rates.

In addition to demographic studies, understanding the influence of climate change on caching species will require experimental work on how specific environmental variables may influence cached food. Such studies could take place in the field (e.g. [139]) or in the laboratory by borrowing many of the approaches used in the field of food science (e.g. [16, 50, 117]). Ultimately, a combination of experimental and demographic studies will be the most rigorous approach for identifying specific mechanisms by which climate change could influence this fascinating group of animals.

\section{Abbreviations}

IPCC: Intergovernmental Panel on Climate Change

\section{Acknowledgements}

We thank two anonymous reviewers for insightful comments that have improved this manuscript.

\section{Funding}

This work was funded by the Natural Sciences and Engineering Research Council of Canada (Discovery Grant to DRN), a University Research Chair (DRN) and an Ontario Graduate Scholarship (AOS).

\section{Availability of data and materials}

Not applicable.

\section{Authors' contributions}

AOS, DS and DRN conceived of the review and contributed to drafting the manuscript. All authors read and approved the final manuscript.

\section{Competing interests}

The authors declare that they have no competing interests.

Consent for publication

Not applicable.

Ethics approval and consent to participate

Not applicable.

\section{Author details}

'Department of Integrative Biology, University of Guelph, 50 Stone Rd East, Guelph, ON N1G 2W1, Canada. ${ }^{2} 1063$ Oxtongue Lake Road, Dwight, ON POA $1 \mathrm{HO}$, Canada.

Received: 4 June 2016 Accepted: 22 November 2016 Published online: 08 December 2016

\section{References}

1. Abdullah N, Nawawi A, Othman I. Fungal spoilage of starch-based foods in relation to its water activity. J Stored Prod Res. 2000;36:47-54.

2. Abro AA, Mahar AR, Mirbahar AA. Improving yield performance of landrace wheat under salinity stress using on-farm seed priming. Pak J Bot. 2009:41:2209-16

3. Alizadeh E, Chapleau N, de Lamballerie M, Le-Bail A. Effect of different freezing processes on the microstructure of Atlantic salmon (Salmo salar) fillets. Innov Food Sci Emerg. 2007;8:493-99.

4. Andersson M, Krebs J. On the evolution of hoarding behaviour. Anim Behav. 1978;26:707-11

5. Arnold W, Heldmaier G, Ortmann S, Pohl H, Ruf T, Steinlechner S. Ambient temperatures in hibernacula and their energetic consequences for alpine marmots Marmota marmota. J Therm Biol. 1991;16:223-26.

6. Aubry KB, Mckelvey KS, Copeland JP. Distribution and broadscale habitat relations of the wolverine in the contiguous United States. J Wildlife Manag. 2007:71:2147-58.

7. Ayala-Zavala JF, Del-Toro-Sanchez L, Alvarez-Parrilla E, Gonzalez-Aguillar GA. High relative humidity in-package of fresh-cut fruits and vegetables: advantage or disadvantage considering microbiological problems and antimicrobial delivering systems? J Food Sci. 2008;73:41-47.

8. Balda RP, Kamil AC. The ecology and evolution of spatial memory in corvids of the southwestern USA: the perplexing pinyon jay. In: Balda RP, Pepperberg IM, Kamil AC, editors. Animal cognition in nature: the convergence of psychology and biology in laboratory and field. 1998. p. 29-64.

9. Baranyi J, Robinson TP, Kaloti A, Mackay BM. Predicting growth of Brochothrix thermosphacta at changing temperature. Int J Food Microbiol. 1995;27:61-75

10. Barrett DM, Lloyd B. Advanced preservation methods and nutrient retention in fruits and vegetables. J Sci Food Agr. 2012;92:7-22.

11. Baskin CC, Baskin JM. Seeds: ecology, biogeography and evolution of dormancy and germination. 2nd ed. Oxford: Elsevier; 1998. 
12. Baskin JM, Baskin CC. Germination ecophysiology of Arenaria glabra, a winter annual of sandstone and granite outcrops of southeastern United States. Am J Bot. 1982;69:973-78.

13. Baskin JM, Baskin CC. Effects of Wedding and drying cycles on the germination of seeds of Cyperus Inflexus. Ecology. 1982;63:248-52.

14. Baskin JM, Baskin CC, Li X. Taxonomy, anatomy and evolution of physical dormancy in seeds. Plant Spec Biol. 2000;15:139-152.

15. Baulu J, Rossi J, Horrocks JA. Food hoarding by a Barbados green monkey (Cercopithecus aethiops sabaeus). Lab Primate Newsletter. 1980; 19:13-14.

16. Boonsumrej S, Chaiwanichsiri S, Tantratian S, Suzuki T, Takai R. Effects of freezing and thawing on the quality of tiger shrimp (Penaeus monodon) frozen by air-blast and cryogenic freezing. J Food Eng. 2007;80:292-99.

17. Borch E, Kant-Muermans ML, Blixt Y. Bacterial spoilage of meat and cured meat products. Int J Food Microbiol. 1996:33:103-20.

18. Both C, Bouwhuis S, Lessells CM, Visser ME. Climate change and population declines in a long-distance migratory bird. Nature. 2006:441:81-83.

19. Both C, Van Asch M, Bijlsma RG, Van Den Burg AB, Visser ME. Climate change and unequal phenological changes across four trophic levels: constraints or adaptations? J Anim Ecol. 2009;78:73-83.

20. Bovill RA, Bew J, Baranyi J. Measurements and predictions of growth for Listeria monocytogenes and Salmonella during fluctuating temperature II. Rapidly changing temperatures. Int J Food Microbiol. 2001;67:131-37.

21. Bovill RA, Bew J, Cook N, D'Agostino M, Wilkinson N, Baranyi J. Predictions of growth for Listeria monocytogenes and Salmonella during fluctuating temperature. Int J Food Microbiol. 2000;59:157-65.

22. Brackett RE. Microbiological consequences of minimally processed fruits and vegetables. J Food Quality. 1987;10:195-206.

23. Brackett RE. Microbiological spoilage and pathogens in minimally processed refrigerated fruits and vegetables. In: Wiley RC, editor. Minimally processed refrigerated fruits \& vegetables. Dordrecht: Springer; 1994. p. 269-312.

24. Brodin A, Lahti K, Lens L, Suhonen J. A northern population of willow tits Parus montanus did not store more food than southern ones. Ornis Fennica. 1996;73:114-18.

25. Cahill AE, Aiello-Lammens ME, Fisher-Reid MC, Hua X, Karanewsky CJ, Ryu HY, et al. How does climate change cause extinction? Proc R Soc B. 2012; doi:10.1098rspb.2012.1890.

26. Carpenter KE, Abrar M, Aeby G, Aronson RB, Banks S, Bruckner A, et al. Onethird of reef-building corals face elevated extinction risk from climate change and local impacts. Science. 2008;321:560-63.

27. Chesemore SG. Ecology of the arctic fox (Alopex lagopus) in North America: a review. In: Fox MW, editor. The wild canids: their systematics, behavioural ecology and evolution. Van Nostrand Rheinhold: New York; 1975. p. 143-63.

28. Copeland JP, McKelvey KS, Aubry KB, Landa A, Persson J, Inman RM, et al. The bioclimatic envelope of the wolverine (Gulo gulo): do climatic constraints limit its geographic distribution? Can J Zool. 2010:88:233-46

29. Covich AP. Optimal use of space by neighbouring central place foragers: when and where to store surplus resources. Adv Behav Econ. 1987;1:249-94.

30. de Kort SR, Clayton NS. An evolutionary perspective on caching by corvids. Proc R Soc London B. 2006;273:417-23.

31. Degange AR, Fitzpatrick JW, Layne JN, Woolfenden GE. Acorn harvesting by Florida scrub jays. Ecology. 1989;70:348-56.

32. Derbyshire R, Strickland D, Norris DR. Experimental evidence and 43 years of monitoring data show that food limits reproduction in a food-caching passerine. Ecology. 2015;96:3005-15.

33. Easterling DR, Meehl GA, Parmesan C, Changnon SA, Karl TR, Mearns LO. Climate extremes: observations, modelling, and impacts. Science. 2000;289:2068-74.

34. Eira MTS, Caldas LS. Seed dormancy and germination as concurrent processes. Rev Bras Fisiol Veg. 2000;12:85-104.

35. Eisenberg JF, Lockhard M. An ecological reconnaissance of the Wilpattu National Park. Ceylon Smithson Contr Zool. 1972;101:1-118.

36. Ekman J, Brodin A, Bylin A. Sklepkovych. Selfish long-term benefits of hoarding in the Siberian jay. Behav Ecol. 1996;7:140-4.

37. Elgmork K. Caching behaviour of brown bears (Ursus arctos). J Mammal. 1982:63:607-12

38. Elliot L. Social behaviour and foraging ecology of the eastern chipmunk (Tamias striatus) in the Adirondack Mountains. Smithson Contr Zool. 1978;265:1-107

39. Eltringham SK. The ecology and conservation of large African mammals. London: Macmillian; 1979.
40. Erb LP, Ray C, Guralnick R. On the generality of a climate-mediated shift in the distribution of the American pika (Ochotona princeps). Ecology. 2011;92: 1730-35.

41. Fernández K, Aspé E, Roeckel M. Scaling up parameters for shelf-life extension of Atlantic Salmon (Salmo salar) fillets using superchilling and modified atmosphere packaging. Food Control. 2010;21:857-62.

42. Finegold L. Molecular and biophysical aspects of adaptation of life to temperatures below the freezing point. Adv Space Res. 1996;18:87-95.

43. Frank LG. Selective predation and seasonal variation in the diet of the fox (Vulpes vulpes) in NE Scotland. J Zool. 1979;189:526-32.

44. Friend AD. Parameterization of a global daily weather generator for terrestria ecosystem and biogeochemical modelling. Ecol Model. 1998;109:121-40.

45. Geiges O. Microbial processes in frozen food. Adv Space Res. 1996;18:109-18.

46. Geneve RL. Some common misconceptions about seed dormancy. Comb Proc Int Plant Prop Soc. 2005:55:9-12.

47. Genkawa T, Uchino T, Inoue A, Tanaka F, Hamanaka D. Development of a low-moisture-content storage system for brown rice: storability at decreased moisture contents. Biosyst Eng. 2008;99:515-22.

48. Gibbons JW, Scott DE, Ryan TJ, Buhlmann KA, Tuberville TD, Metts BS, et al. The Global Decline of Reptiles, Déjà Vu Amphibians Reptile species are declining on a global scale. Six significant threats to reptile populations are habitat loss and degradation, introduced invasive species, environmental pollution, disease, unsustainable use, and global climate change. Bioscience. 2000;50:653-66.

49. Gibson AM, Bratchell N, Roberts TA. Predicting microbial growth: growth responses of salmonellae in a laboratory medium as affected by $\mathrm{pH}$, sodium chloride and storage temperature. Int J Food Microbiol. 1988;6:155-78.

50. Gormley R, Walshe T, Hussey K, Butler F. The effect of fluctuating vs. constant frozen storage temperature regimes on some quality parameters of selected food products. LWT-Food Sci Technol. 2002;35:190-200.

51. Gram L, Dalgaard P. Fish spoilage bacteria-problems and solutions. Curr Opin Biotech. 2002;13:262-66.

52. Gram L, Huss HH. Microbiological spoilage of fish and fish products. Int J Food Microbiol. 1996;33:121-37.

53. Grass JE, Gould LH, Mahon BE. Epidemiology of foodbourne disease outbreaks caused by Clostridium perfringens. United States Foodborne Pathog Dis. 2013;10:131-36.

54. Grøtan V, Sæther B, Engen S, van Balen JH, Perdeck AC, Visser ME. Spatial and temporal variation in the relative contribution of density dependence, climate variation and migration to fluctuations in the size of great tit populations. J Anim Ecol. 2009;78:447-59.

55. Hansen E, Juncher D, Henckel P, Karlsson A, Bertelsen G, Skibsted LH. Oxidative stability of chilled pork chops following long-term frozen storage. Meat Sci. 2004:68:479-84.

56. Hardy GA. Squirrel cache of fungi. Can Field Nat. 1949;63:86-87.

57. Honacki JA, Kinman KE, Koeppl JW. Mammal species of the world: a taxonomic and geographic reference. Baltimore; John Hopkins University Press; 1982.

58. Hughes L. Biological consequences of global warming: Is the signal already apparent? Trends Ecol Evol. 2000;15:56-61.

59. Hughey $\mathrm{VL}$, Johnson EA. Antimicrobial activity of lysozyme against bacteria involved in food spoilage and food-borne disease. Appl Environ Microb. 1987:53:2165-70.

60. Huis in't Veld JHJ. Microbial and biochemical spoilage of foods: an overview. Int J Food Microbiol. 1996:33:1-18.

61. Immerseel FV, Buck JD, Pasmans F, Huyghebaert G, Haesebrouck F, Ducatelle R. Clostridium perfringens in poultry: an emerging threat for animal and public health. Avian Pathol. 2004:33:537-49.

62. Inman RM, Magoun AJ, Persson J, Mattisson J. The wolverine's niche: linking reproductive chronology, caching, competition and climate. J Mammal. 2012;93:634-44

63. Intergovernmental Panel on Climate Change. Climate Change 2014 Synthesis Report. In: Pachauri RK, Meyer LA, editors. Contribution of Working Groups I, II and III to the Fifth Assessment Report of the Intergovernmental Panel on Climate Change. Geneva, Switzerland: IPCC; 2014. 151

64. Isengard HD. Water content, one of the most important properties of food. Food Control. 2001;12:395-400

65. Jacobs LF, Linman ER. Grey squirrels remember the locations of buried nuts. Anim Behav. 1991;41:103-10.

66. Jansson C, Ekman J, von Bromssen A. Winter mortality and food supply in tits Parus spp. Oikos. 1981;37:313-22.

67. Jenkins JR, Devenport LD. Seed preparation diminishes cache loss in least chipmunks. J Mammal. 2014;95:276-83. 
68. Jeong J, Kim G, Yang H, Joo S. Effect of freeze-thaw cycles on physicochemical properties and color stability of beef Semimembranosus muscle. Food Res Int. 2011:44:3222-28.

69. Kaale LD, Eikevik TM, Rustad T, Kolsaker K. Superchilling of food: A review. J Food Eng. 2011;107:141-46.

70. Kamil AC, Balda RP. Cache recovery and spatial memory in Clark's Nutcrackers (Nucifraga columbiana). J Exp Psychol Anim B. 1985;11:95-111.

71. Kawamichi T. Hay territory rank of pikas (Ochotona princeps). J Mamm. 1976; 57:133-48.

72. Keenan SW, Engel AS, Elsey RM. The alligator gut microbiome and implications for archosaur symbioses. Sci Rep. 2013;3:2877.

73. Keith DA, Akçakaya HR, Thuiller W, Midgley GF, Pearson RG, Phillips SJ, et al. Predicting extinction risks under climate change: coupling stochastic population models with dynamic bioclimatic habitat models. Biol Letters. 2008;4:560-63.

74. Kell DB, Kaprelyants AS, Weichart DH, Harwood CR, Barer MR. Viability and activity in readily culturable bacteria: a review and discussion of the practical issues. Anton Leeuw Int J Microb. 1998;73:169-87.

75. Kilham L. Covering of stores by white-breasted and red-breasted nuthatches. Condor. 1974;76:108-09.

76. Kimball JS, Running SW, Nemani R. An improved method for estimating surface humidity from daily minimum temperature. Agr icForest Meteorol. 1997;85:87-98

77. Klamt M, Thompson R, Davis J. Early response of the platypus to climate warming. Global Change Biol. 2011;17:3011-18.

78. Körner C. Alpine plant life: functional plant ecology of high mountain ecosystems. Berlin: Springer Science \& Business Media; 2003.

79. Korpimacki E. Prey caching of breeding Tengmalm's owls Aegolius funereus as a buffer against temporary food shortage. Ibis. 1987;129:499-510.

80. Kostic AD, Chun E, Robertson L, Glickman JN, Gallini CA, Michaud M, et al. Fusobacterium nucleatum potentiates intestinal tumorigenesis and modulates the tumor-immune microenvironment. Cell Host Microbe. 2013;14:207-15.

81. Lahti K, Koivula K, Rytkönen S, Mustonen T, Welling P, Pravosudov W, et al Social influences on food caching in willow tits: a field experiment. Behav Ecol. 1998;9:122-29.

82. Lawrence MG. The relationship between relative humidity and the dewpoint temperature in moist air: a simple conversion and applications. Bull Am Meteorol Soc. 2005:86:225-33.

83. Leaver LA, Daly M. Food caching and differential cache pilferage: a field study of coexistence of sympatric kangaroo rats and pocket mice. Oecologia. 2001;128:577-84.

84. Leskovar DI, Esensee V, Belefant-Miller H. Pericarp, leachate and carbohydrate involvement in thermoinhibition of germinating spinach seeds. J Am Soc Hortic Sci. 1999;124:301-06.

85. Ley RE, Hamady M, Lozupone C, Turnbaugh PJ, Ramey RR, Bircher JS, et al. Evolution of mammals and their gut microbes. Science. 2008;320:1647-51.

86. Leygonie C, Britz TJ, Hoffman LC. Impact of freezing and thawing on the quality of meat: review. Meat Sci. 2012;91:93-98.

87. Li B, Sun DW. Novel methods for rapid freezing and thawing of foods: a review. J Food Eng. 2002;54:175-82

88. Ligon JD. The biology of the elf owl, Micrathene whitneyi. Misc Publ Mus Zool Univ Mich. 1968;136:1-70.

89. Lush WM, Groves RH, Kaye PE. Presowing hydration-dehydration treatments in relation to seed germination and early seedling growth of wheat and ryegrass. Funct Plant Biol. 1981;8:409-25

90. Macdonald DW, Brown L, Yerli S, Canbolat A. Behavior of red foxes, Vulpes vulpes, caching eggs of loggerhead turtles, Caretta caretta. J Mammal. 1994; 75:985-88.

91. Mackey, B.M. Lethal and sublethal effects of refrigeration, freezing and freeze-drying on micro-organisms. In: Andrew MHE, Russell AD, editors. The revival of injured microbes. London: Academic Press; 1984. p. 45-75.

92. MacRoberts MH, MacRoberts BR. Social organization and behaviour of the acorn woodpecker in central coastal California. Ornithol Monogr. 1976;21:1-115.

93. Makoto K, Kajimoto T, Koyama L, Kudo G, Shibata H, Yanai Y, Cornelissen JHC. Winter climate change in plant-soil systems: summary of recent findings and future perspectives. Ecol Res. 2014;29:593-606.

94. Mann M, Amman C, Bradley R, Briffa K, Jones P, Osborn T, et al. On past temperatures and anomalous late-20 th -century warmth. Eos T Am Geophys Un. 2003;84:256.

95. Martin IG. Venom of the short-tailed shrew (Blarina brevicauda) as an insect immobilizing agent. J Mammal. 1981;62:189-92.
96. Mathlouthi M. Water content, water activity, water structure and the stability of foodstuffs. Food Control. 2001;12:409-17.

97. Matos E. Effect of harvesting stress and storage conditions on protein degradation in fillets of farmed gilthead seabream (Sparus aurata): a differential scanning calorimetry study. Food Chem. 2011;126:270-76.

98. Mazur P. Limits to life at low temperatures and at reduced water contents and water activities. In: Limits of Life. Dordrecht: Springer. 1980. p. 137-59.

99. McCarty JP. Ecological consequences of recent climate change. Conserv Biol. 2001;15:320-31.

100. McCord CM, Cardoza JE. Bobcat and lynx. In: Feldhamer GA, Thompson BC, Chapman JA, editors. Wild mammals of North America: biology, management, economics. 1982. p. 728-66.

101. McKenzie VJ, Bowers RM, Fierer N, Knight R, Lauber CL. Co-habiting amphibian species harbor unique skin bacterial communities in wild populations. ISME J. 2012;6:588-96.

102. McMeekin TA, Ross T. Shelf life prediction: status and future possibilities. Int J Food Microbiol. 1996;33:65-83.

103. Miles CA, Mayer Z, Morley MJ, Houska M. Estimating the initial freezing point of foods from compositional data. Int J Food Sci Tech. 1997;32:389-400.

104. Moller H. Foods and foraging behaviour of red (Sciurus vulgaris) and grey (Sciurus carolinensis) squirrels. Mammal Rev. 1983;13:81-98.

105. Moreno J, Lundberg A, Carlson A. Hoarding of individual nuthatches Sitta europea and marsh tits Parus palustris. Holarctic Ecol. 1981;4:263-69.

106. Morrison SF, Hik DS. Demographic analysis of a declining pika Ochotona collaris population: linking survival to broad-scale climate patterns via spring snowmelt patterns. J Anim Ecol. 2007;76:899-907.

107. Mossel DAA, Corry JE, Strujik CB, Baird RM. Essentials of the microbiology of foods: a textbook for advanced studies. Chichester: John Wiley \& Sons; 1995.

108. Nakazawa T. A perspective on match/mismatch of phenology in community contexts. Oikos. 2012;121:489-95.

109. Nishimoto RK, McCarty LB. Fluctuating temperature and light influence seed germination of goosegrass (Eleusine indica). Weed Sci. 1997:45:426-29.

110. Norris DR, Flockhart DT, Strickland D. Contrasting patterns of survival and dispersal in multiple habitats reveal an ecological trap in a food-caching bird. Oecologia. 2013;173:827-35.

111. O'Reilly C, De Atrip N. Seed moisture content during chilling and heat stress effects after chilling on the germination of common alder and downy birch seeds. Silva Fenn. 2007:41:235.

112. Ohishi I, Sakaguchi G, Riemann H, Behymer D, Hurvell B. Antibodies to Clostridium botulinum toxins in free-living birds and mammals. J Wildlife Dis. 1979;15:3-9.

113. Oliver JD. The viable but nonculturable state in bacteria. J Microbiol. 2005; 43:93-100.

114. Oliver JD, Bockian R. In vivo resuscitation, and virulence towards mice, of viable but nonculturable cells of Vibrio vulnificus. Appl Environ Microb. 1995; 61:2620-23

115. Oliver JD, McDougald D, Barrett T, Glover LA, Prosser Jl. Effect of temperature and plasmid carriage on nonculturability in organisms targeted for release. FEMS Microbiol Ecol. 1995:17:229-37.

116. Petit DR, Petit LJ, Petit KE. Winter caching ecology of deciduous woodland birds and adaptations for protection of stored food. Condor. 1989:91:766-76.

117. Phimolsiripol Y, Siripatrawan U, Tulyathan V, Cleland DJ. Effects of freezing and temperature fluctuations during frozen storage on frozen dough and bread quality. J Food Eng. 2008;84:48-56.

118. Pill WG, Bender RH, Pie AC, Marvel JK, Veacock EE. Responses of six wildflower species to seed matric priming. J Environ Hortic. 2000;18:160-65.

119. Pin C, Baranyi J, Sutherland JP. Validating predictive models of food spoilage organisms. J Appl Microbiol. 1999;87:491-99.

120. Post $E$, Forchhammer MC. Climate change reduces reproductive success of an Arctic herbivore through trophic mismatch. Philos T Roy Soc B. 2008;363:2367-73.

121. Post E, Forchhammer MC, Bret-Harte MS, Callaghan TV, Christensen TR, Elberling B, et al. Ecological dynamics across the Arctic associated with recent climate change. Science. 2009:325:1355-58.

122. Potter NN, Hotchkiss JH. Food Science. New York: Springer Science \& Business Media; 2012.

123. Pravosudov W. On seasonality in food-storing behaviour in parids: do we know the whole story? Anim Behav. 2006;71:1455-60.

124. Pravosudov W, Clayton NS. A test of the adaptive specialization hypothesis: population differences in caching, memory, and the hippocampus in blackcapped chickadees (Poecile atricapilla). Behav Neurosci. 2002;116:515-22. 
125. Pravosudov W, Clayton NS. Effects of demanding foraging conditions on cache retrieval accuracy in food-caching mountain chickadees (Poecile gambeli). Proc R Soc Lond B Bio. 2001;268:363-68.

126. Pritchard HW, Manger KR, Prendergast FG. Changes in Trifolium arvense seed quality following alternating temperature treatment using liquid nitrogen. Ann Bot London. 1988;62:1-11.

127. Probert RJ. The role of temperature in the regulation of seed dormancy and germination. In: Fenner $M$, editor. Seeds: the ecology of regeneration in plant communities. 2000. p. 261-92

128. Qi J, Li C, Chen Y, Gao F, Xu X, Zhou G. Changes in meat quality of ovine longissimus dorsi muscle in response to repeated freeze and thaw. Meat Sci. 2012;92:619-26.

129. Ragaert P, Devlieghere F, Debevere J. Role of microbiological and physiological spoilage mechanisms during storage of minimally processed vegetables. Postharvest Biol Tec. 2007:44:185-94.

130. Rahman I, Shahamat M, Kirchrnan PA, Russek-Cohen F, Colwell RR. Methionine uptake and cytopathogenicity of viable but nonculturable Shigella dysenteriae type 1. Appl Environ Microb. 2009;60:3573-78.

131. Ratkowsky DA, Olley J, McMeekin TA, Ball A. Relationship between temperature and growth rate of bacterial cultures. J Appl Bacteriol. 1982;149:1-5.

132. Ratti C. Hot air and freeze-drying of high-value foods: a review. J Food Eng. 2001:49:311-19

133. Reichman OJ, Fattaey A, Fattaey K. Management of sterile and moldy seeds by a dessert rodent. Anim Behav. 1986;34:221-25.

134. Reichman OJ, Wicklow DT, Rebar C. Ecological and mycological characteristics of caches in the mounds of Dipodomys spectabilis. J Mammal. 1985;66:643-51.

135. Rich T, Trentlage B. Caching of long-horned beetles (Cerambycidae: Prionus integer) by the burrowing owl. Murrelet. 1983;64:25-26.

136. Roggenbuck M, Schnell IB, Blom N, Baelum J, Bertelsen MF, Sicheritz-Ponten T, et al. The microbiome of New World vultures. Nat Commun. 2014;5:1-7.

137. Roos Y, Karel M. Differential scanning calorimetry study of phase transitions affecting the quality of dehydrated materials. Biotechnol Progr. 1990;6:159-63.

138. Schaller GB. The deer and the tiger. Chicago: University of Chicago Press; 1967.

139. Scheley T, Strickland D, Norris DR. Linking the availability of cached food to climate change: an experimental test of the hoard-rot hypothesis. Can J Zool. 2015;93:411-19.

140. Sechley T, Strickland D, Norris DR. Causes and consequences of pre-laying weight gain in a food-caching bird that breeds in late winter. J Avian Biol. 2014;45:85-93.

141. Shank CC. Territory size, energetics and breeding strategy in the Corvidae. Am Nat. 1986;128:642-52.

142. Sherry DF. Food storing in the paridae. Wilson Bull. 1989;101:289-304.

143. Shimono $Y$, Kudo G. Comparisons of germination traits of alpine plants between fellfield and snowbed habitats. Ecol Res. 2005:20:189-97.

144. Shin JS, Raymer P, Kim W. Environmental factors influencing germination in seeded seashore paspalum. Hortscience. 2006:41:1330-31.

145. Smith CC, Reichman OJ. The evolution of food caching by birds and mammals. Ann Rev Ecol Syst. 1984;15:329-51.

146. Snead E, Hendrickson GO. Food habits of the badger in lowa. J Mammal. 1942:23:380-91.

147. Soja A, Tchebakova N, French N, Flannigan MD, Shugart HH, Stocks BJ, et al. Climate-induced boreal forest change: predictions versus current observations. Glob Planet Chang. 2007;56:274-96.

148. Sriket P, Benjakul S, Visessanguan W, Kijroongrojana K. Comparative studies on the effect of the freeze-thawing process on the physicochemical properties and microstructures of black tiger shrimp (Penaeus mondon) and white shrimp (Penaeus vannamei) muscle. Food Chem. 2007;104:113-21.

149. Soltani A, Tigabu M, Odén PC. Alleviation of physiological dormancy in oriental beechnuts with cold stratification at controlled and unrestricted hydration. Seed Sci Technol. 2005;33:283-92.

150. Srinivasan S, Xiong YL, Blanchard SP, Tidwell JH. Physicochemical changes in prawns (Machrobrachium rosenbergii) subjected to multiple freeze-thaw cycles. J Food Sci. 1997;62:123-27.

151. Stannard CJ, Williams AP, Gibbs PA. Temperature/growth relationship for psychrotrophic food-spoilage bacteria. J Food Microbiol. 1985;2:115-22.

152. Stocks BJ, Fosberg MA, Lynham TJ, Mearns L, Wotton BM, Yang Q, et al. Climate change and forest fire potential in Russian and Canadian boreal forests. Climate Change. 1998;38:1-13.

153. Stothart MR, Bobbie CB, Schulte-Hostedde Al, Boonstra R, Palme R, Mykytczuk NC, et al. Stress and the microbiome: linking glucocorticoids to bacterial community dynamics in wild red squirrels. Biol Letters. 2016; doi: 10.1098/rsbl.2015.0875

154. Strickland D, Ouellet H. Gray jay (Perisoreus canadensis). In: The Birds of North America Online. Cornell Lab of Ornithology. 2011. http://bna.birds. cornell.edu/bna/species/040. Accessed 14 Apr 2016.

155. Strickland D, Kielstra B, Norris DR. Experimental evidence for a novel mechanism driving variation in habitat quality in a food-caching bird. Oecologia. 2011;167:943-50.

156. Sturm M, Holmgren M, König M, Morris K. The thermal conductivity of seasonal snow. J Glaciol. 1997;43:26-41.

157. Sulkava P, Huhta V. Effects of hard frost and freeze-thaw cycles on decomposer communities and $\mathrm{N}$ mineralization in boreal forest soil. Appl Soil Ecol. 2003:22:225-39.

158. Sulkava S, Nyholm ES. Mushroom stores as a winter food of the red squirrel, Sciurus vulgaris, in northern Finland. Aquilo Ser Zool. 1987;25:1-8.

159. Taylorsen RB. Water stress-induced germination of giant foxtail (Sertaria faberi). Weed Sci. 1986;34:871-75.

160. Tomback DF. How nutcrackers find their seed stores. Condor; 1980. p. 10-19.

161. Tournas VH. Spoilage of vegetable crops by bacteria and fungi and related health hazards. Crit Rev Microbiol. 2005;31:33-44.

162. Trenberth KE, Shea DJ. Relationships between precipitation and surface temperature. Geophys Res Lett. 2005; doi: 10.1029/2005GL022760.

163. Van Assche JA, Debucquoy KL, Rommens WA. Seasonal cycles in the germination capacity of buried seeds of some Leguminosae (Fabaceae). New Phytol. 2003;158:315-23.

164. Vander Wall SB. Food Hoarding in Animals. Chicago: University of Chicago Press; 1990.

165. Vander Wall SB, Balda RP. Coadaptations of the Clark's nutcracker and the pinon pine for efficient seed harvest and dispersal. Ecol Mongr. 1977:47:89-111.

166. Vander Wall SB, Smith KG. Cache protecting behaviour of food-hoarding animals. Foraging Behaviour. In: Kamil AC, Krebs JR, Pulliam RH, editors. Foraging behaviour. 1987. p. 611-44.

167. Vander Wall SB, Briggs JS, Jenkins SH, Kuhn KM, Thayer TC, Beck MJ. Do food-hoarding animals have a cache recovery advantage? Determining recovery of stored food. Anim Behav. 2006;72:189-97.

168. Varner J, Horns JJ, Lambert MS, Westberg E, Ruff JS, Wolfenberger K, et al. Plastic pikas: behavioural flexibility in low-elevation pikas (Ochtona princeps). Behav Process. 2016:125:63-71.

169. Vleeshouwers LM, Bouwmeester HJ, Karssen CM. Redefining seed dormancy: an attempt to integrate physiology and ecology. J Ecol. 1995:83:1031-37.

170. Wagner JR, Añón MC. Effect of freezing rate on the denaturation of myo-fibrillar proteins. Int J Food Sci Tech. 1985;20:735-44.

171. Waite TA, Strickland D. Climate change and the demographic demise of a hoarding bird living on the edge. P Roy Soc Lond B Bio. 2006;273:2809-13.

172. Walther GR. Community and ecosystem responses to recent climate change. Philos T Roy Soc B. 2010;365:2019-24.

173. Wang $X$, Piao S, Ciais $P$, Li J, Friedlingstein $P$, Koven $C$, et al. Spring temperature change and its implication in the change of vegetation growth in North America from 1982 to 2006. PNAS. 2011;108:1240-50.

174. Wesche AM, Gurtler JB, Marks BP, Ryser ET. Stress, sublethal injury, resuscitation and virulence of bacterial foodborne pathogens. J Food Protect. 2009;72:1121-38.

175. Winder M, Schindler DE. Climate change uncouples trophic interactions in an aquatic ecosystem. Ecology. 2004;85:2100-06.

176. Whelan S, Strickland D, Morand-Ferron J, Norris DR. Male experience buffers female laying date plasticity in a winter-breeding, food-storing passerine. Anim Behav. 2016;121:61-70

177. Wolf $P$, Oliver JD. Temperature effects on the viable but nonculturable state of Vibrio vulnificus. FEMS Microbiol Lett. 1992:101:33-39.

178. Wolf SG, Snyder MA, Sydeman WJ, Doak DF, Croll DA. Predicting population conseuqences of ocean climate change for an ecosystem sentinel, the seabird Cassin's auklet. Glob Change Biol. 2010;16:1923-35.

179. Woodrey MS. Caching behaviour in free-ranging white-breasted nuthatches: the effects of social dominance. Ornis Scand. 1991:22:160-66.

180. Xia XF, Kong BH, Liu Q, Liu J. Physicochemical change and protein oxidation in porcine longissimus dorsi as influenced by different freeze-thaw cycles. Meat Sci. 2009:83:239-45.

181. Xia $X$, Kong $B$, Xiong $Y$, Ren $Y$. Decreased gelling and emulsifying properties of myofibrillar protein from repeatedly frozen-thawed porcine longissimus muscle are due to protein denaturation and susceptibility to aggregation. Meat Sci. 2010;85:481-86. 
182. Zhou G, Xu X, Liu Y. Preservation technologies for fresh meat: a review. Meat Sci. 2010;86:119-28.

183. Zuur-Isler D. Germinating behaviour and early life phase of some species from alpine serpentine soils. Berlin Geobot Inst ETH. 1982;49:76-107.

184. Zwietering MH, De Koos JT, Hasenack BE, De Wit JC, Van't Riet K. Modeling of bacterial growth as a function of temperature. Appl Environ Microbiol. 1991;57:1094-101.

Submit your next manuscript to BioMed Central and we will help you at every step:

- We accept pre-submission inquiries

- Our selector tool helps you to find the most relevant journal

- We provide round the clock customer support

- Convenient online submission

- Thorough peer review

- Inclusion in PubMed and all major indexing services

- Maximum visibility for your research

Submit your manuscript at www.biomedcentral.com/submit 